\begin{tabular}{|c|c|c|}
\hline \multirow{2}{*}{ ENGINEERING CHANGE NOTICE } & & 1. ECN 657320 \\
\hline & Page 1 of 2 & $\begin{array}{l}\text { Proj. } \\
\text { ECN }\end{array}$ \\
\hline
\end{tabular}

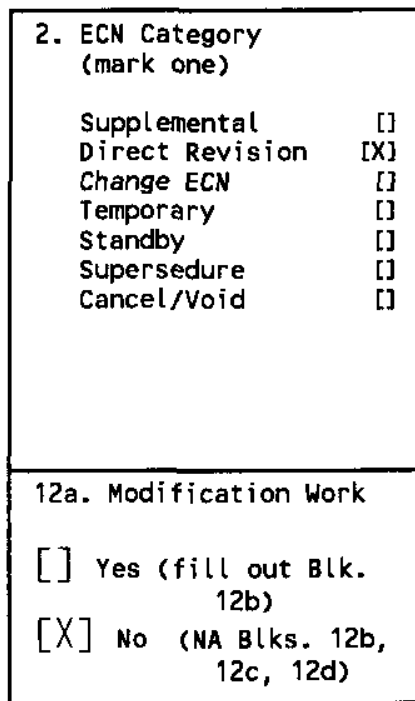

13a. Description of Change Complete revision.
3. Originator's Name, Organization, MSIN, and Telephone No.

Andrew M. Templeton, Data

Development and Interpretation, R2-12, 373-5589

6. Project Title/No./Work Order No.

Tank 241-AZ-101

9. Document Numbers Changed by this ECN (includes sheet no. and rev.) RPP-5534, Rev. 1

\begin{tabular}{|l|l|}
\hline $\begin{array}{l}\text { 4. USQ Required? } \\
{[] \text { Yes }[X] \text { No }}\end{array}$ & 0 \\
\hline $\begin{array}{c}\text { 7. Bldg./Sys./Fac. No. } \\
241-A Z-101\end{array}$ & 8 \\
\hline $\begin{array}{l}\text { 10. Related ECN No(s). } \\
\text { ECN-657303 }\end{array}$ & 11
\end{tabular}

5. Date

04/07/00

8. Approval Designator QE

11. Related PO No.

$N / A$ 12b. Work Package $12 \mathrm{c}$. Modification Work Complete No.

$\mathrm{N} / \mathrm{A}$

$$
N / A
$$

12d. Restored to Original Condition (Temp. or Standby ECN only) $\mathrm{N} / \mathrm{A}$

Design Authority/Cog. Engineer Signature \& Date

Design Authority/Cog. Engineer Signature \& Date $[X]$ No

13b. Design Baseline Document?

[] Yes [X] No




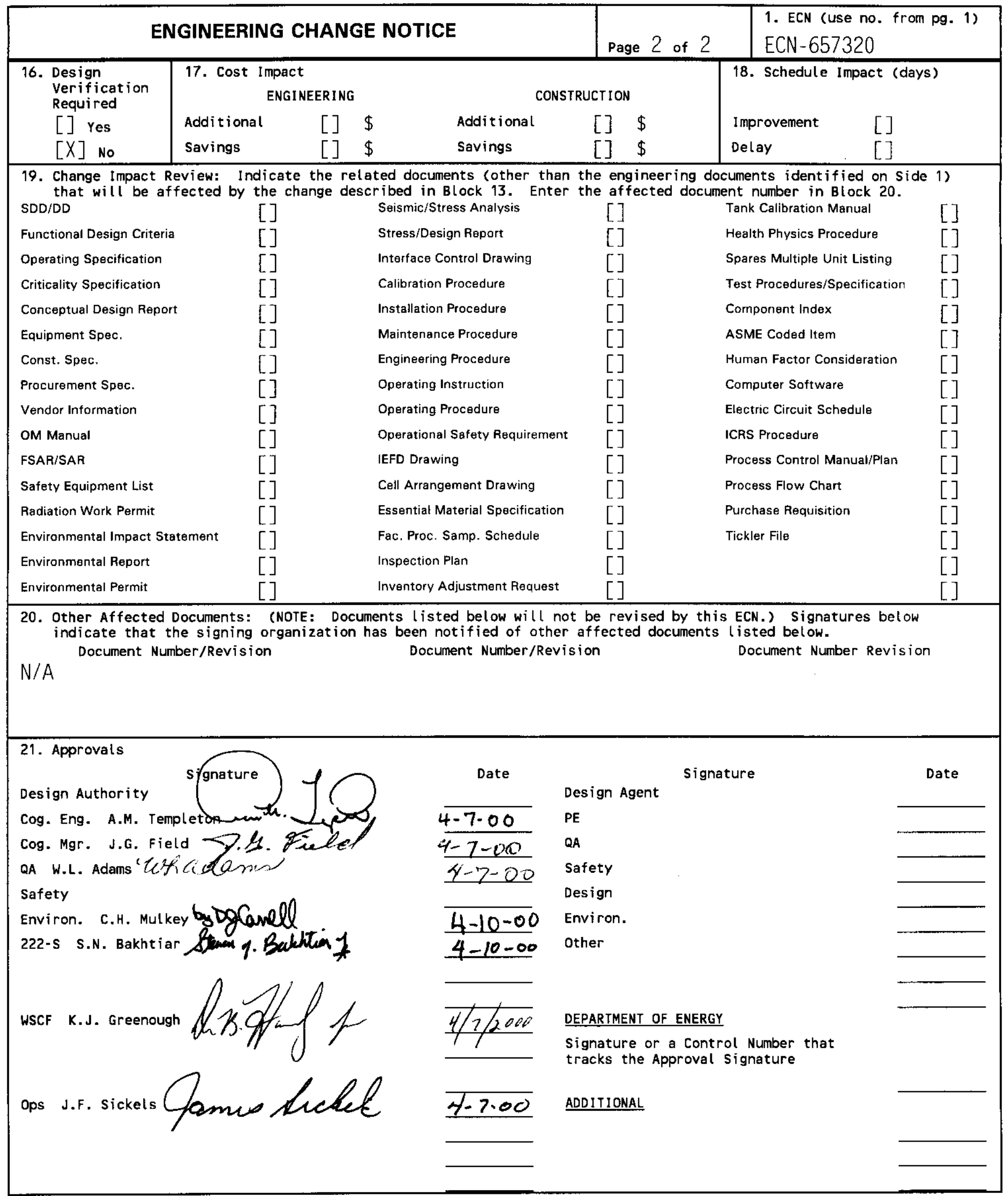




\section{Tank 241-AZ-101 Mixer Pump Test Vapor Sampling and Analysis Plan}

Andrew M. Templeton

CH2M HILL Hanford Group. Inc., Richland, WA 99352

U.S. Department of Energy Contract DE-AC06-96RL13200

EDT/ECN: $\quad$ ECN-657320

Org Code: $74 \mathrm{~B} 20$

B\&R Code: EW 3120074 Total Pages: 37

Key Words: Tank 241-AZ-101, 241-AZ-101, Tank AZ-101, AZ-101, AZ Farm, Mixer Pump Test Vapor Sammpling, Vapor Sampling, Vapor, Sampling, Analysis

Abstract: N/A

TRADEMARK DISCLAIMER. Reference herein to any specific comercial product, process, or service by trade name, trademark, manufacturer, or otherwise, does not necessarily constitute or imply its endorsenent, recommendation, or favoring by the United States Government or any agency thereof or its contractors or subcontractors.

Printed in the United States of America. To obtain copies of this document, contact: Document Control Services, P.O. Box 950, Mailstop H6-08, Richland WA 99352, Phone (509) 372-2420; Fax (509) 376-4989.
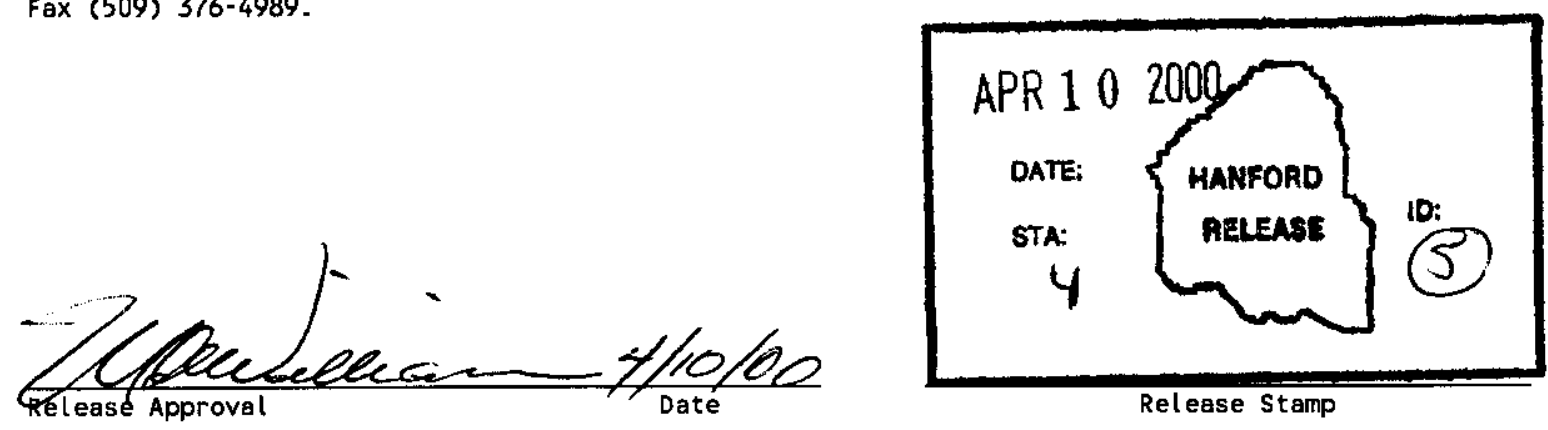

Approved for Public Release 


\section{RECORD OF REVISION}

(1) Document Number

RPP-5534

(2) Title

Tank 241-AZ-101 Mixer Pump Test Vapor Sampling and Analysis Plan

CHANGE CONTROL RECORD

(3) Revision 1 (4) Description of Change - Replace, Add, and Delete Pages

0 (7) Initia11y released $01 / 31 / 00$ on EDT627271.

1 RS

Incorporate per ECN-657303. Incorporate per ECh- 657320.

Authorized for Release

(5) Cog. Engr. (6) Cog. Mgr. Date

\begin{tabular}{l|l}
\hline A.M. & J.G. Field
\end{tabular}

Templeton

A.M.

J.G. Field

Templetor

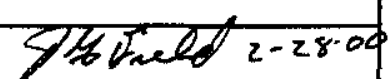

Ro2
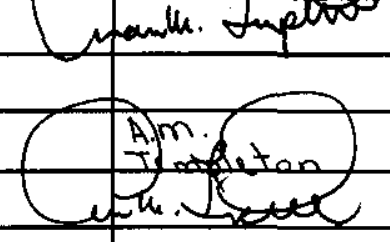

J.G. Field

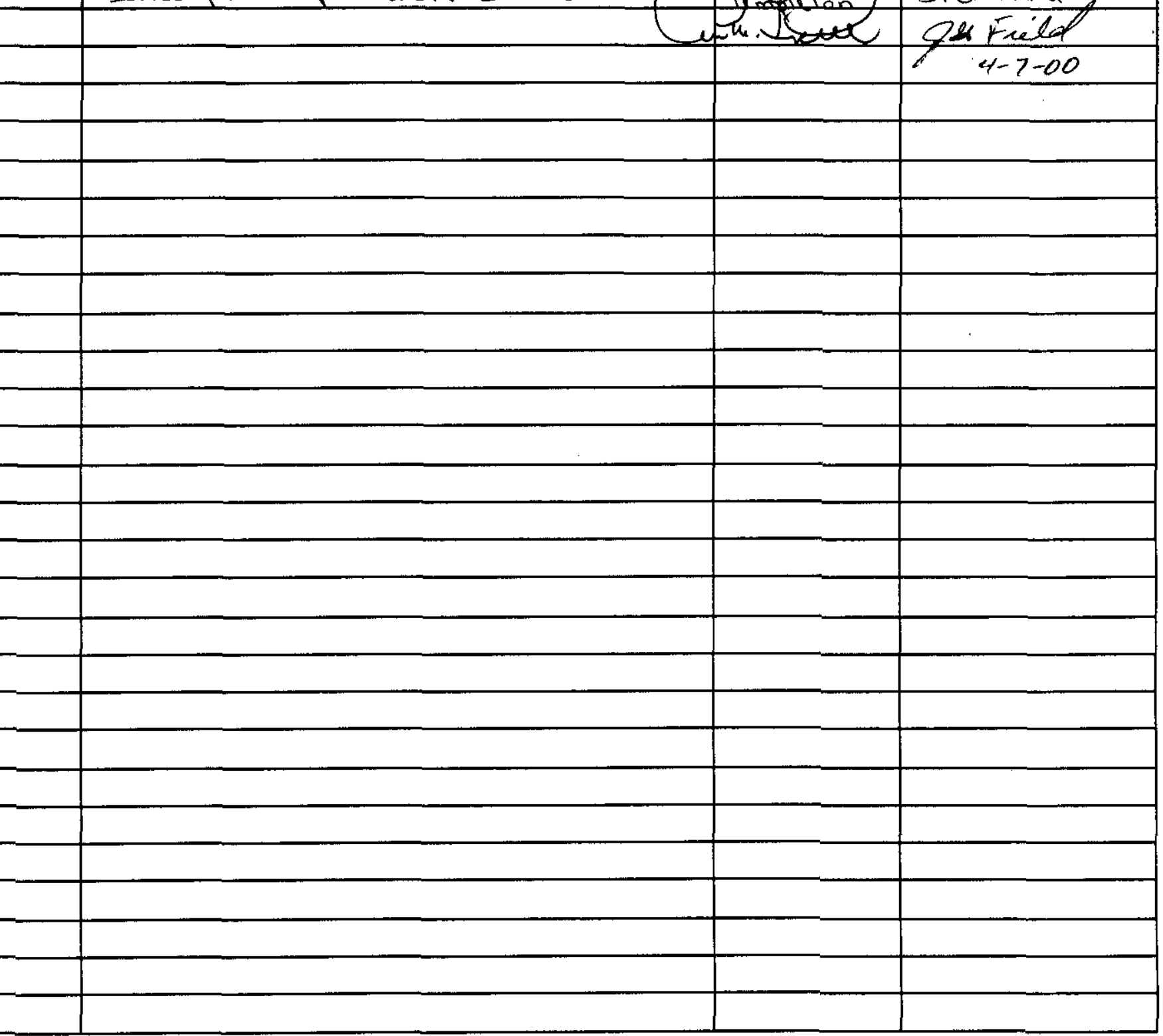


RPP-5534

Revision 2

\title{
Tank 241-AZ-101 Mixer Pump Test Vapor Sampling and Analysis Plan
}

\author{
A. M. Templeton \\ CH2M HILL Hanford Group, Inc.
}

Date Published

April 2000

Prepared for the U.S. Department of Energy

Office of River Protection 
RPP-5534 Rev. 2

\section{TABLE OF CONTENTS}

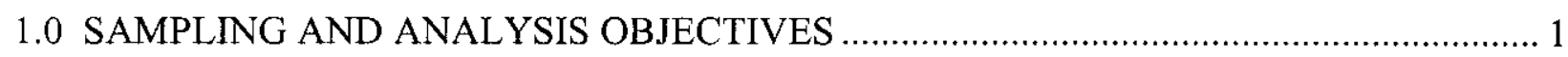

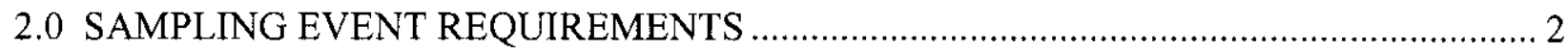

2.1 TYPE 4 IN-SITU VAPOR SAMPLER SYSTEM DESCRIPTION ............................. 2

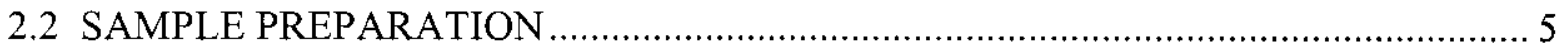

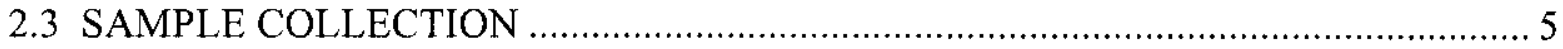

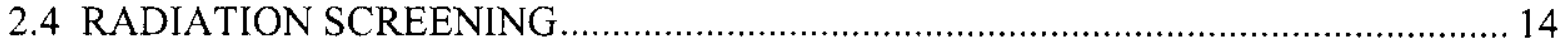

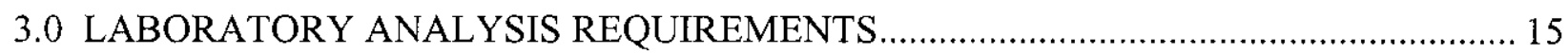

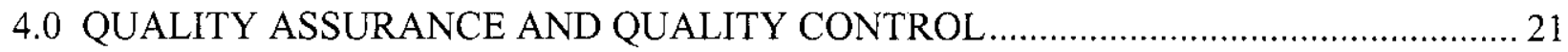

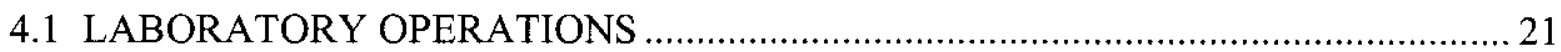

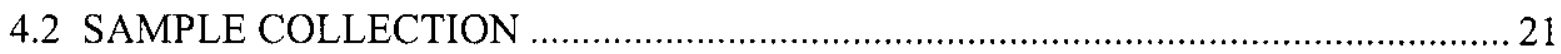

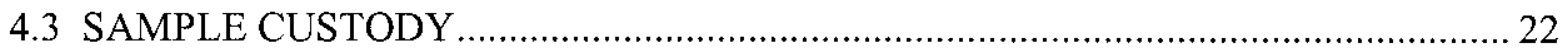

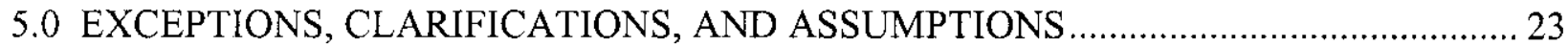

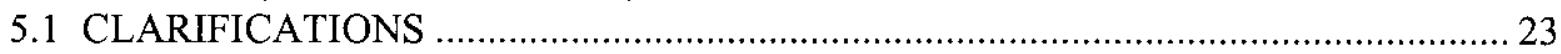

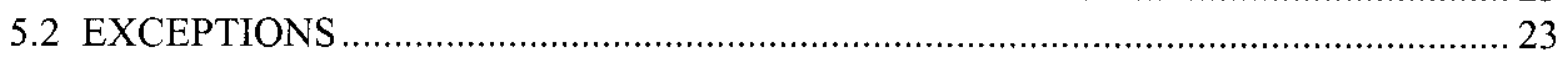

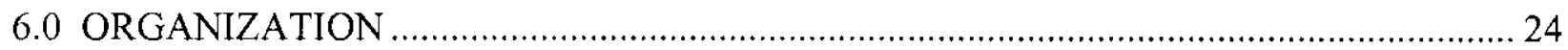

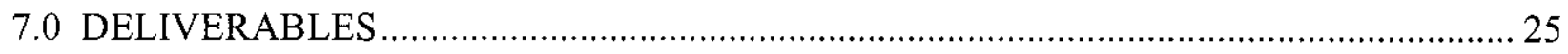

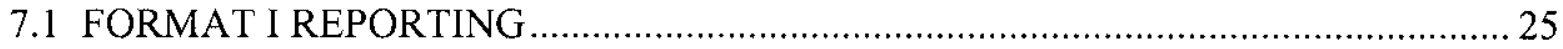

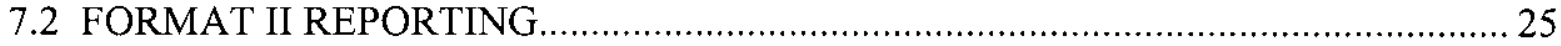

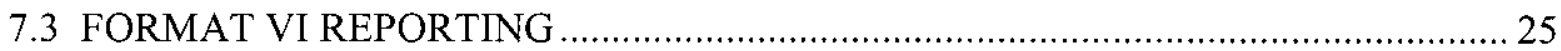

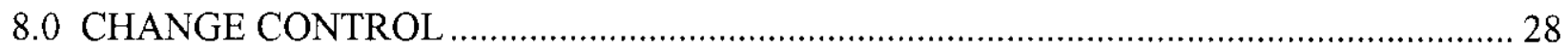

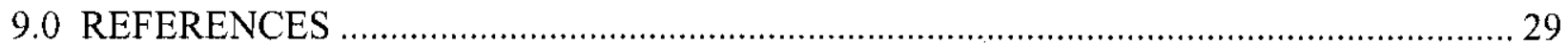

\section{APPENDICES}

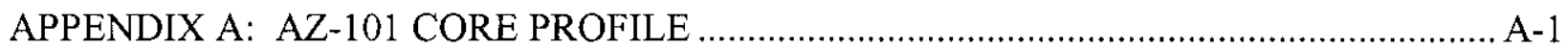




\section{LIST OF TABLES}

Table 2-1. Sample Preparation Procedures .................................................................... 5

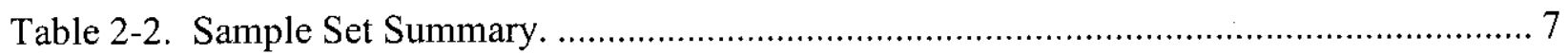

Table 2-3. List of Samples and Activities for 241-AZ-101 Headspace ................................. 7

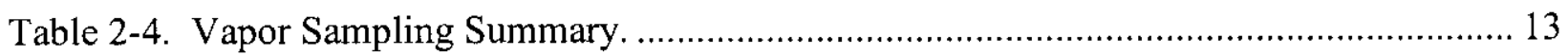

Table 3-1. Chemical and Radiological Analytical Requirements...................................... 16

Table 3-2. Vapor Analytes and Quantitation Limits..................................................... 17

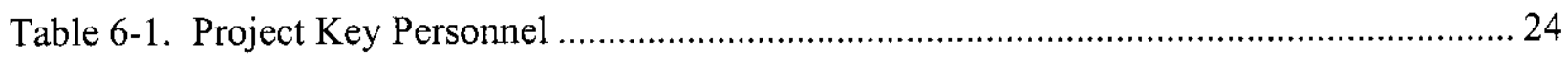

Table 7-1. Data Package Required Elements............................................................. 26

\section{LIST OF FIGURES}

Figure 2-1. Type 4 In-Situ Vapor Sampling System ................................................. 3

Figure 2-2. Typical Sampling Head Arrangement of Sorption Tubes and Particle Filters........... 4 
RPP-5534 Rev. 2

\section{LIST OF ABBREVIATIONS}

$\begin{array}{ll}\text { CAS } & \text { Chemical Abstracts Service } \\ \text { CHG } & \text { CH2M HILL Hanford Group, Inc. } \\ \text { CPO } & \text { Characterization Project Operations } \\ \text { DBP } & \text { dibutyl phosphate } \\ \text { FID } & \text { flame ionization detector } \\ \text { ft } & \text { feet } \\ \text { GC/MS } & \text { gas chromatography/mass spectrometry } \\ \text { GC/TCD } & \text { gas chromatography/thermal conductivity detector } \\ \text { IC } & \text { ion chromatography } \\ \text { IDLH } & \text { immediately dangerous to life and health } \\ \text { ISVS } & \text { In-situ vapor sampler } \\ \text { kgal } & \text { kilogallon } \\ \text { kL } & \text { kiloliter } \\ \text { L } & \text { liter } \\ \text { L/min } & \text { liters per minute } \\ \text { LFL } & \text { lower flammability limit } \\ \text { m } & \text { meter } \\ \text { mg/m } & \text { milligrams per cubic meters } \\ \text { mL } & \text { milliliters } \\ \text { mL/min } & \text { milliliters per minute } \\ \text { min } & \text { minutes } \\ \text { Mgal } & \text { million gallons } \\ \text { N/A } & \text { not applicable or not available } \\ \text { NHC } & \text { Numatec Hanford Corporation } \\ \text { OVM } & \text { organic vapor monitor } \\ \text { ppbv } & \text { parts per billion by volume } \\ \text { ppm } & \text { parts per million } \\ \text { ppmv } & \text { parts per million by volume } \\ \text { PUF } & \text { polyurethane foam } \\ \text { QA } & \text { quality assurance } \\ \text { QC } & \text { quality control } \\ \text { rpm } & \text { revolutions per minute } \\ \text { RPP } & \text { River Protection Project } \\ \text { SAP } & \text { Sampling and Analysis Plan } \\ \text { STT } & \text { sorbent tube train } \\ \text { TBP } & \text { tributyl phosphate } \\ \text { TNMOC } & \text { Total non-methane organic compound } \\ \text { TST } & \text { triple sorbent trap } \\ \text { VOC } & \text { Volatile Organic Compounds } \\ \text { VPRQL } & \text { Vapor Program Required Quantitation Limit } \\ \text { WSCF } & \text { Waste Sampling and Characterization Facility } \\ \text { \% } & \end{array}$




\subsection{SAMPLING AND ANALYSIS OBJECTIVES}

This sampling and analysis plan (SAP) identifies characterization objectives pertaining to sample collection, laboratory analytical evaluation, and reporting requirements for vapor samples obtained during the operation of mixer pumps in tank 241-AZ-101. The primary purpose of the mixer pump test (MPT) is to demonstrate that the two 300 horsepower mixer pumps installed in tank 241-AZ-101 can mobilize the settled sludge so that it can be retrieved for treatment and vitrification. Sampling will be performed in accordance with Tank 241-AZ-101 Mixer Pump Test Data Quality Objective (Banning 1999) and Data Quality Objectives for Regulatory Requirements for Hazardous and Radioactive Air Emissions Sampling and Analysis (Mulkey 1999). The sampling will verify if current air emission estimates used in the permit application are correct and provide information for future air permit applications.

Tank 241-AZ-101 has been selected for the first full-scale demonstration testing of a retrieval system. Tank 241-AZ-101 is located in the 241-AZ Tank Farm in the 200 East Area. The tank is a $3,785,400-\mathrm{L}$ (1-Mgal), 23-m (75-ft) diameter double-shell underground storage tank. As of January 2, 2000, the surface level ENRAF' indicated that tank 241-AZ-101 contained a total waste volume of approximately $3,213 \mathrm{~kL}(849 \mathrm{kgal})$. Based upon sludge level measurements taken in August 1997, tank 241-AZ-101 contains 3,024 kL (799 kgal) supernate and $178 \mathrm{~kL}$ (47 kgal) sludge. This waste volume is equivalent to 7.8 meters (308.6 inches) of waste as measured from the inside bottom of the tank. A physical profile based on waste sampling information is provided in Appendix A. Previous characterization data indicate the tank contains primarily aging waste (Hodgson 1995).

A series of tests will be conducted in which the two mixer pumps in tanks 241-AZ-101 will be operated in fixed and oscillating modes and at different speeds. Vapor samples will be obtained to determine constituents that may be present in the headspace of tank $241-\mathrm{AZ}-101$ as the waste is disturbed by mixer pump operations. During the testing, vapor samples will be obtained from the headspace of tank 241 -AZ-101 via riser $15 \mathrm{G}$ or $15 \mathrm{~L}$ using the Type 4 in-situ vapor sampler (ISVS) cart. Riser 24C, the unused port on the standard hydrogen monitoring system (SHMS) will provide as a backup. Results will be used to provide the waste feed delivery program with environmental air permitting data for tank waste disturbing activities. Because of radiological issues, some samples may be extracted at the 222-S Laboratory if contaminated. All SUMMA ${ }^{\mathrm{TM}}$ canister and some Triple Sorbent Trap (TSTs) and Sorbent Tube Trains (STTs) samples sent to the Waste Sampling and Characterization Facility (WSCF) laboratory will be filtered for particulates. It is recognized that this may remove some organic compounds. Duplicate unfiltered STT samples will also be collected and either analyzed or extracted at 222-S laboratories if contaminated. Duplicate unfiltered TST samples will also be collected and extracted at 222-S laboratories if contaminated.

The following sections provide the general methodology and procedures to be used in the preparation, retrieval, transport, analysis, and reporting of results from vapor samples retrieved during the mixer pump testing.

\footnotetext{
' ENRAF is a trademark of the ENRAF Corporation, Houston, Texas.
} 


\subsection{SAMPLING EVENT REQUIREMENTS}

The mixer pumps will be operated during a series of tests. Sample sets will be taken prior to mixer pump operation, during the initial startup of pump \#1 in fixed mode, when pump \#1 is first operated in oscillating mode, and when both pumps are operated in oscillating mode at maximum speed $(1200 \mathrm{rpm})$. The exact sample time during these activities, except the baseline, will be determined by headspace monitoring using an organic vapor monitor (OVM). A minimum set of four sample sets will be collected even if no organics are detected with the OVM. If a release of organics is detected, then additional optional sets of samples will be collected as directed by Process Engineering. Before and after sample collection, the SUMMA ${ }^{2}$ canister line on the Type 4 ISVS will be monitored continuously with an OVM. Riser $24 \mathrm{C}$ will be monitored continuously during the mixer pump test under a separate industrial hygiene (IH) sampling/monitoring plan (Fogg 2000). This IH plan will also include headspace monitoring for lower flammability limit (LFL), Notice of Construction (NOC) required stack monitoring and personnel monitoring. Tank vapor will also be monitored via the Standard Hydrogen Monitoring System (SHMS) cabinet. Details of the SHMS is included in the Data Collection Plan for AZ-101 Mixer Pump Test, HNF-3839.

\subsection{TYPE 4 IN-SITU VAPOR SAMPLER SYSTEM DESCRIPTION}

The Type 4 ISVS system includes three major assemblies: sampling cart, tube bundle, and sampling head. The ISVS cart (Figure 2-1), consists of an instrumentation cabinet, vacuum air pump components, a manifold, and various valves, flow meters, flow totalizers, rotameters, and filters mounted on a hand truck. A tube bundle connects the cart with the sampling head and provides a separate sampling tube for a SUMMA ${ }^{\mathrm{TM}}$ canister sampler. The sampling head shown in Figure 2-2 (Figure 2-2 is considered a typical arrangement of sorption tubes and filters) contains particulate filters, tritium-trap filters, multiple sorption tubes, and a thermocouple. The selection and arrangement of the sorption tubes and filters is dependent upon the vapor sampling needs identified in the Data Quality Objective (Mulkey 1999) document and this sampling analysis plan (SAP) that define tank sampling requirements. The tube-bundle is used to lower the sampling head through a riser into a tank's vapor space. Thermocouples on the sampling head and in the cart's gas manifold are used to measure vapor temperature (Reich 2000).

After sampling is completed, the tube bundle with the sampling head is removed from the tank. The sample head is disconnected from the tube bundle and the ends of the sorption and filter channels are capped. The sampling head is bagged out with protective plastic for shipment to the laboratory (Reich 2000).

\footnotetext{
${ }^{2}$ SUMMA is a trademark of Molectrics of Ohio.
} 


\section{RPP-5534 Rev. 2}

Figure 2-1. Type 4 In-Situ Vapor Sampling System

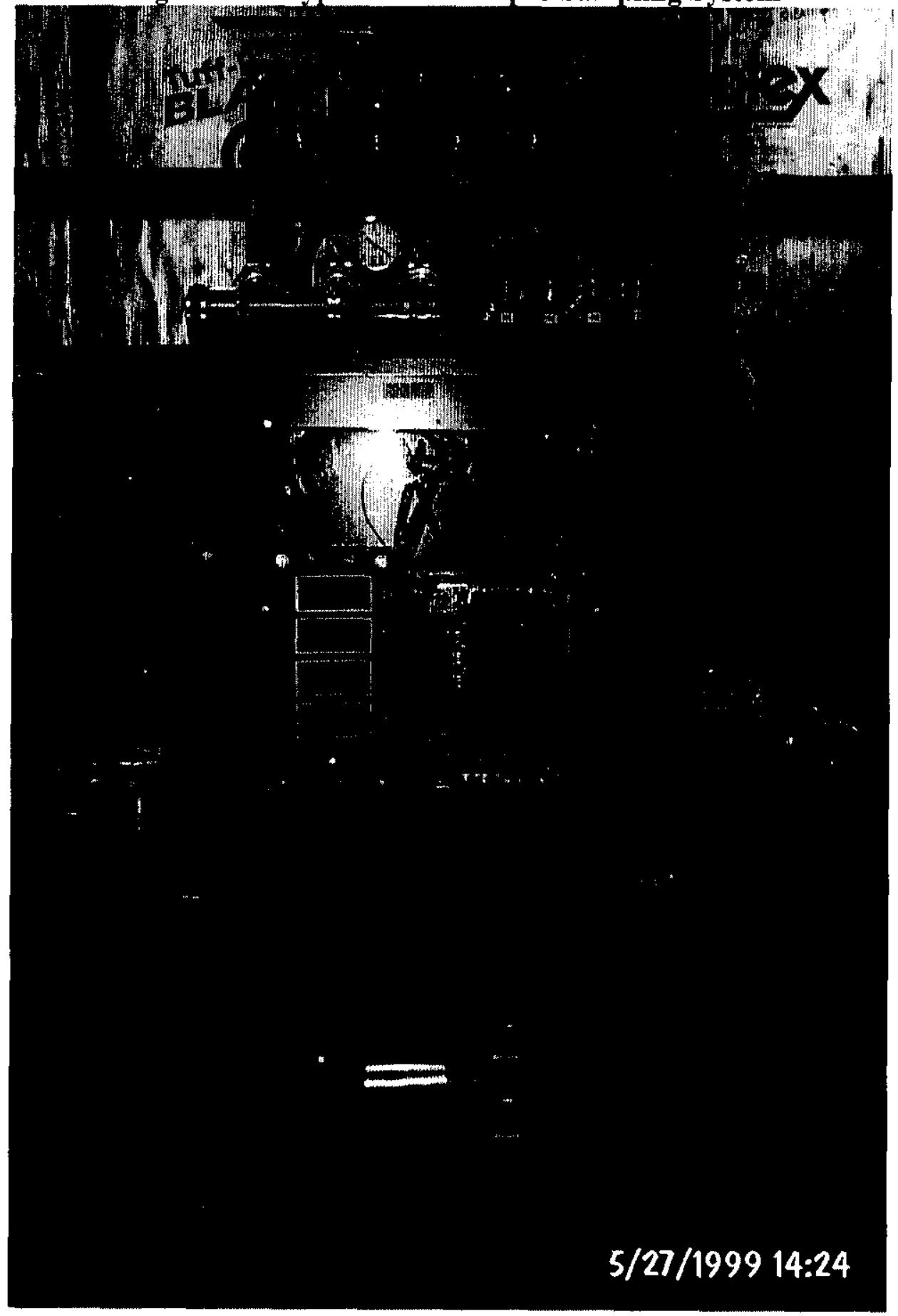


RPP-5534 Rev. 2

Figure 2-2. Typical Sampling Head Arrangement of Sorption Tubes and Particle Filters.

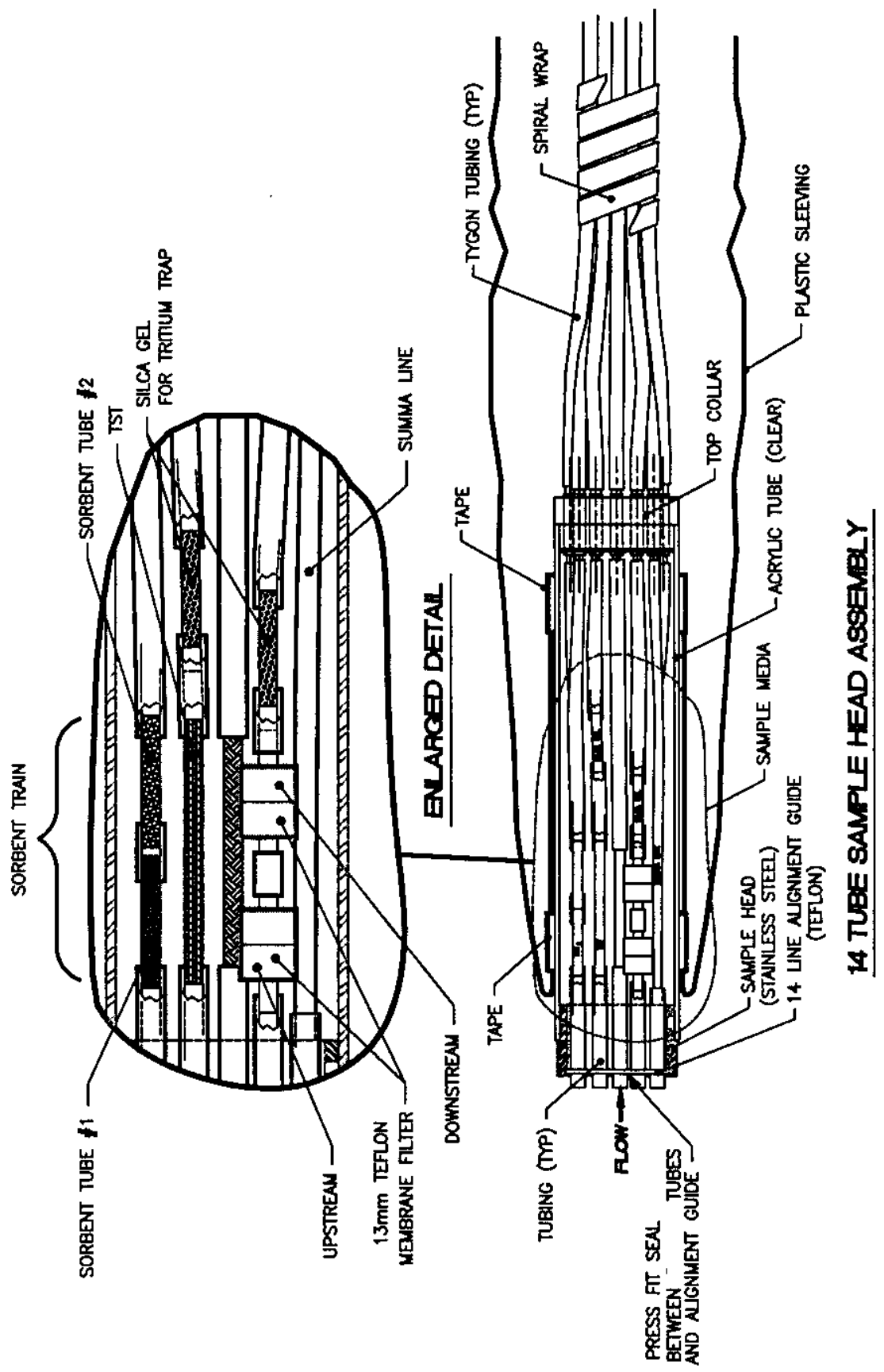




\subsection{SAMPLE PREPARATION}

SUMMA ${ }^{\mathrm{TM}}$ canister samples, triple sorbent trap (TST) samples, sorbent tube train (STT) samples, and polyurethane foam (PUF) samples will be collected and sent to the laboratory for analysis; field and trip blanks will accompany the samples. The analytical laboratory for this sampling event is Fluor Hanford (FH) WSCF and 222-S laboratories. Particulate filter samples will be collected for radiation screening of the samples. WSCF shall prepare the SUMMA ${ }^{\mathrm{TM}}$ canisters, TSTs, STTs, and PUFs for sample collection. WSCF shall also prepare the particulate filter assemblies. Each particulate filter assembly shall contain two particulate filters mounted in series. Sample preparation procedures are listed in Table 2-1. The sample head assembly shall be assembled in accordance with drawing H-2-825301, current revision, In Situ Sample Head Assembly and Details and documented per Section 4.3, "Sample Custody."

Table 2-1. Sample Preparation Procedures

\begin{tabular}{|c|c|c|}
\hline Sample container & Organization & Preparation Procedure \\
\hline SUMMA $^{\mathrm{TM}}$ canister & WSCF & LO-080-406 \\
\hline TST & WSCF & LA-549-403 \\
\hline STT & WSCF & LA-549-402 \\
\hline PUF & WSCF & $\mathrm{N} / \mathrm{A}$ \\
\hline Particulate filter assembly & WSCF & $\mathrm{N} / \mathrm{A}$ \\
\hline
\end{tabular}

\subsection{SAMPLE COLLECTION}

Characterization Project Operations (CPO) will be responsible for the collection of all SUMMA TM canister samples, particulate filter, TST, STT, and PUF samples using the type 4 cart. Samples will be collected in accordance with procedure TO-080-627, Vapor Sampling of Waste Tank Using In-Situ Vapor Sampling (ISVS) System. If the type 4 cart is not available, the non-electrical vapor sampling system (NEVS) will be used in accordance with procedure LO-080-400, Vapor Sampling Using the Non-Electrical Vapor Sampling System (NEVS). CPO baseline sampling activities will be performed in accordance with work package ES-00-00030.

Tubing used in the collection of vapor samples must be stainless steel, or Teflon ${ }^{3}$, and the length of the tubing used should be minimized. No C-flex or tygon tubing can be used upstream of the sample containers.

The samples shall include the following quality control (QC) samples: one each SUMMA ${ }^{\mathrm{TM}}$, TST, STT, and PUF ambient air field blank; and one each STT, and PUF trip blank and up to 8 TST trip blanks. The trip and field blanks are to accompany the vapor samples to the laboratory.

${ }^{3}$ Teflon is a registered trademark of I. E. DuPont De Nemours and Company 
For specific information concerning sample and blank handling, custody, and transport, refer to the requirements in Section 4.0.

Characterization Project Operations (CPO) shall record (or calculate) the following information for each sample collected as appropriate: sample number, start and stop times for the collection of each sample, sample volumes, and any anomalous sampling conditions. The volatile organic compounds (VOC) reading will be collected at the beginning and at the end of the collection of each sample set. The samples shall be sealed in plastic, stored and shipped in a cooler at a nominal 4 degrees Celsius except the SUMMA ${ }^{\mathrm{TM}}$ canisters and the particulate filters.

Particulate filter samples should be shipped by CPO to WSCF for radiation screening analysis within one calendar day and must be shipped within three calendar days. The remaining filtered SUMMA $^{\mathrm{TM}}$ samples shall be stored by CPO until radiation screening is completed and the samples can be released for analysis. Upon completion of the radiation screening and release of the samples, the samples shall be transferred by CPO to WSCF for analysis.

All other filtered and unfiltered samples should be shipped directly to 222-S within one calendar day and must be shipped within three calendar days.

Table 2-3 provides the sequence of sampling activities for the blanks and the tank 241-AZ-101 headspace air samples. The samples are summarized in Table 2-4. SUMMA ${ }^{\mathrm{TM}} \mathrm{s}$ and three of the four TST samples per set and one STT per set will be collected through particulate filters. The particulate filter samples shall be collected upstream of the SUMMA ${ }^{\text {TM }}$ canisters, TSTs, and STTs.

At a minimum, the following samples are required from the 241-AZ-101 headspace:

- One set of samples before the pumps are started (baseline samples).

- One set of samples after pump \#1 is initially started and operating in a fixed mode at minimum speed (700 rpm) and at the direction of Process Engineering (one optional set of samples may also be collected at the direction of Process Engineering).

- One set when pump \#1 is first operated in oscillating mode (two optional sets of samples may also be collected at the direction of Process Engineering).

- One set when both pumps are operated at maximum speed in oscillating mode (one optional set of samples may also be collected at the direction of Process Engineering).

The sample sets and optional sample sets are summarized in Table 2-2. After collecting the set of samples for each event, the tube bundle and head will be withdrawn from the tank. The sample head will be replaced with the optional sample head and re-installed in the tank headspace. If a release is detected in the headspace, as indicated by the OVM, or has already occurred, the optional sample sets will be collected. The collection of these samples will be determined and directed by Process Engineering. 
Table 2-2. Sample Set Summary.

\begin{tabular}{|l|l|l|l|}
\hline \multicolumn{1}{|c|}{ Sample Container } & \multicolumn{1}{c|}{ Number } & \multicolumn{1}{c|}{ Analyte } & \multicolumn{1}{c|}{ Procedure } \\
\hline SUMMA & 1 & Organic Speciation & LA-523-404 \\
\hline SUMMA & 1 & Permanent Gases & LA-523-409 \\
\hline SUMMA & 1 & $\begin{array}{l}\text { Total Nonmethane } \\
\text { Hydrocarbons }\end{array}$ & LA-523-407 \\
\hline STT & 3 & $\begin{array}{l}\text { NH } \text { Oxides of } \\
\text { Nitrogen }\end{array}$ & TBD \\
\hline TST & 4 & Organic Speciation & LA-523-408 \\
\hline PUF & 2 & TBP, DPB, SVOCs & TBD \\
\hline Particulate Filter & 1 & $\begin{array}{l}\text { Total Alpha, Total } \\
\text { Beta and Gamma } \\
\text { Energy }\end{array}$ & TBD \\
\hline
\end{tabular}

$\mathrm{TBD}=$ to be determined

The sampling activities of CPO shall be coordinated so that, for each set of samples, the SUMMA $^{\text {TM }}$ canisters, TSTs, STTs, and PUFs are obtained at about the same time.

Any decisions, observations, or deviations affecting this SAP shall be documented in controlled notebooks, work packages, and justified in the deliverable report.

Table 2-3. List of Samples and Activities for 241-AZ-101 Headspace (6 sheets).

\begin{tabular}{|c|c|c|c|c|c|}
\hline $\begin{array}{l}\text { Sample } \\
\text { Code }\end{array}$ & Sample/Activity Description & $\begin{array}{l}\text { Sample } \\
\text { Probe } \\
\text { Line }\end{array}$ & $\begin{array}{l}\text { Sampler Position } \\
\text { During Collection }\end{array}$ & $\begin{array}{l}\text { Sample } \\
\text { Volume }^{2}\end{array}$ & $\begin{array}{c}\text { Sample } \\
\text { Duration or } \\
\text { Flow Rate }\end{array}$ \\
\hline \multicolumn{6}{|c|}{ Leak Test } \\
\hline--- & $\begin{array}{l}\text { Perform leak test of Type IV Cart } \\
\text { or NEVS }\end{array}$ & $\mathrm{N} / \mathrm{A}$ & N/A & N/A & N/A \\
\hline \multicolumn{6}{|c|}{ Ambient Samples } \\
\hline 001 & $\begin{array}{l}\text { Collect upwind ambient } \\
\text { SUMMA }{ }^{\mathrm{TM}} \text { before pump is started. }\end{array}$ & $\mathrm{N} / \mathrm{A}$ & AZ Tank farm & $6 \mathrm{~L}$ & $2 \mathrm{~min}$ \\
\hline 002 & $\begin{array}{l}\text { Collect system SUMMA }{ }^{\mathrm{TM}} \text { before } \\
\text { pump is started. }\end{array}$ & $\mathrm{N} / \mathrm{A}$ & AZ Tank farm & $6 \mathrm{~L}$ & $2 \mathrm{~min}$ \\
\hline 003 & $\begin{array}{l}\text { Collect ambient TST before pump } \\
\text { is started }\end{array}$ & 1 & AZ Tank farm & $0.4 \mathrm{~L}$ & $\begin{array}{c}50-200 \\
\mathrm{~mL} / \mathrm{min}\end{array}$ \\
\hline 004 & $\begin{array}{l}\text { Collect ambient STT before pump } \\
\text { is started }\end{array}$ & 2 & AZ Tank farm & $8 \mathrm{~L}$ & $\begin{array}{l}300-500 \\
\mathrm{~mL} / \mathrm{min}\end{array}$ \\
\hline 005 & $\begin{array}{l}\text { Collect ambient PUF before pump } \\
\text { is started }\end{array}$ & 3 & AZ Tank farm & $25 \mathrm{~L}$ & $2.5-5 \mathrm{~L} / \mathrm{min}$ \\
\hline
\end{tabular}


Table 2-3. List of Samples and Activities for 241-AZ-101 Headspace (6 sheets).

\begin{tabular}{|c|c|c|c|c|c|}
\hline $\begin{array}{l}\text { Sample } \\
\text { Code }\end{array}$ & Sample/Activity Description & $\begin{array}{l}\text { Sample } \\
\text { Probe } \\
\text { Line }\end{array}$ & $\begin{array}{l}\text { Sampler Position } \\
\text { During Collection }\end{array}$ & $\begin{array}{l}\text { Sample } \\
\text { Volume }^{2}\end{array}$ & $\begin{array}{l}\text { Sample } \\
\text { Duration or } \\
\text { Flow Rate }^{1}\end{array}$ \\
\hline \multicolumn{6}{|c|}{ Baseline headspace samples } \\
\hline $\mathrm{N} / \mathrm{A}$ & $\begin{array}{l}\text { Purge SUMMA }{ }^{\mathrm{TM}} \text { line with tank } \\
\text { vapor. }\end{array}$ & N/A & $\begin{array}{l}\text { 241-AZ-101 } \\
\text { Headspace }\end{array}$ & $5 \mathrm{~L}$ & $1.0-5.0 \mathrm{~L} / \mathrm{min}$ \\
\hline 006 & $\begin{array}{l}\text { Collect baseline SUMMA } \\
\text { canister/particulate filter before } \\
\text { pump is started }\end{array}$ & $\mathrm{N} / \mathrm{A}$ & $\begin{array}{l}\text { 241-AZ-101 } \\
\text { Headspace }\end{array}$ & $6 \mathrm{~L}$ & $2 \mathrm{~min}$ \\
\hline 007 & $\begin{array}{l}\text { Collect baseline SUMMA } \\
\text { canister/particulate filter before } \\
\text { pump is started }\end{array}$ & $\mathrm{N} / \mathrm{A}$ & $\begin{array}{l}\text { 241-AZ-101 } \\
\text { Headspace }\end{array}$ & $6 \mathrm{~L}$ & $2 \mathrm{~min}$ \\
\hline 008 & $\begin{array}{l}\text { Collect baseline SUMMA } \\
\text { canister/particulate filter before } \\
\text { pump is started }\end{array}$ & $\mathrm{N} / \mathrm{A}$ & $\begin{array}{l}\text { 241-AZ-101 } \\
\text { Headspace }\end{array}$ & $6 \mathrm{~L}$ & $2 \min$ \\
\hline 009 & $\begin{array}{l}\text { Collect particulate filter and tritium } \\
\text { trap sample before pump is started }\end{array}$ & 1 & $\begin{array}{l}\text { 241-AZ-101 } \\
\text { Headspace }\end{array}$ & $1 \mathrm{~L}$ & $\begin{array}{l}50-200 \\
\mathrm{~mL} / \mathrm{min}\end{array}$ \\
\hline 010 & $\begin{array}{l}\text { Collect baseline TST before pump } \\
\text { is started }\end{array}$ & 2 & $\begin{array}{c}241-\mathrm{AZ}-101 \\
\text { Headspace }\end{array}$ & $0.4 \mathrm{~L}$ & $\begin{array}{l}50-200 \\
\mathrm{~mL} / \mathrm{min}\end{array}$ \\
\hline 011 & $\begin{array}{l}\text { Collect baseline filtered TST before } \\
\text { pump is started }\end{array}$ & 3 & $\begin{array}{l}\text { 241-AZ-101 } \\
\text { Headspace }\end{array}$ & $0.4 \mathrm{~L}$ & $\begin{array}{l}50-200 \\
\mathrm{~mL} / \mathrm{min}\end{array}$ \\
\hline 012 & $\begin{array}{l}\text { Collect baseline filtered TST before } \\
\text { pump is started }\end{array}$ & 4 & $\begin{array}{l}\text { 241-AZ-101 } \\
\text { Headspace }\end{array}$ & $0.4 \mathrm{~L}$ & $\begin{array}{c}50-200 \\
\mathrm{~mL} / \mathrm{min}\end{array}$ \\
\hline 013 & $\begin{array}{l}\text { Collect baseline filtered TST before } \\
\text { pump is started }\end{array}$ & 5 & $\begin{array}{l}\text { 241-AZ-101 } \\
\text { Headspace }\end{array}$ & $0.4 \mathrm{~L}$ & $\begin{array}{l}50-200 \\
\mathrm{~mL} / \mathrm{min}\end{array}$ \\
\hline 014 & $\begin{array}{l}\text { Collect baseline STT before pump } \\
\text { is started }\end{array}$ & 6 & $\begin{array}{l}\text { 241-AZ-101 } \\
\text { Headspace }\end{array}$ & $4 \mathrm{~L}$ & $\begin{array}{l}300-500 \\
\mathrm{~mL} / \mathrm{min}\end{array}$ \\
\hline 015 & $\begin{array}{l}\text { Collect baseline STT before pump } \\
\text { is started }\end{array}$ & 7 & $\begin{array}{l}\text { 241-AZ-101 } \\
\text { Headspace }\end{array}$ & $8 \mathrm{~L}$ & $\begin{array}{l}300-500 \\
\mathrm{~mL} / \mathrm{min}\end{array}$ \\
\hline 016 & $\begin{array}{l}\text { Collect baseline filtered STT before } \\
\text { pump is started }\end{array}$ & 8 & $\begin{array}{l}\text { 241-AZ-101 } \\
\text { Headspace }\end{array}$ & $8 \mathrm{~L}$ & $\begin{array}{l}300-500 \\
\mathrm{~mL} / \mathrm{min}\end{array}$ \\
\hline 017 & $\begin{array}{l}\text { Collect baseline PUF trap before } \\
\text { pump is started }\end{array}$ & 9 & $\begin{array}{l}\text { 241-AZ-101 } \\
\text { Headspace }\end{array}$ & $25 \mathrm{~L}$ & $2.5-5 \mathrm{~L} / \mathrm{min}$ \\
\hline 018 & $\begin{array}{l}\text { Collect baseline PUF trap before } \\
\text { pump is started }\end{array}$ & 10 & $\begin{array}{c}\text { 241-AZ-101 } \\
\text { Headspace }\end{array}$ & $25 \mathrm{~L}$ & $2.5-5 \mathrm{~L} / \mathrm{min}$ \\
\hline \multicolumn{6}{|c|}{ Sampling during mixer pump operation } \\
\hline \multicolumn{6}{|c|}{ Collect sample set \#2 during initial operation of Pump \#1 at $700 \mathrm{rpm}$} \\
\hline $\mathrm{N} / \mathrm{A}$ & $\begin{array}{l}\text { Purge SUMMA }{ }^{\mathrm{TM}} \text { line with tank } \\
\text { vapor. }\end{array}$ & N/A & $\begin{array}{c}241-\mathrm{AZ}-101 \\
\text { Headspace }\end{array}$ & $5 \mathrm{~L}$ & $1.0-5.0 \mathrm{~L} / \mathrm{min}$ \\
\hline 019 & $\begin{array}{l}\text { Collect SUMMA } \\
\text { canister/particulate filter }\end{array}$ & N/A & $\begin{array}{c}\text { 241-AZ-101 } \\
\text { Headspace } \\
\end{array}$ & $6 \mathrm{~L}$ & $2 \mathrm{~min}$ \\
\hline 020 & $\begin{array}{l}\text { Collect SUMMA } \\
\text { canister/particulate filter }\end{array}$ & $\mathrm{N} / \mathrm{A}$ & $\begin{array}{l}\text { 241-AZ-101 } \\
\text { Headspace }\end{array}$ & $6 \mathrm{~L}$ & $2 \mathrm{~min}$ \\
\hline 021 & $\begin{array}{l}\text { Collect SUMMA } \\
\text { canister/particulate filter }\end{array}$ & $\mathrm{N} / \mathrm{A}$ & $\begin{array}{c}241-\mathrm{AZ}-101 \\
\text { Headspace }\end{array}$ & $6 \mathrm{~L}$ & $2 \min$ \\
\hline
\end{tabular}


RPP-5534 Rev. 2

Table 2-3. List of Samples and Activities for 241-AZ-101 Headspace (6 sheets).

\begin{tabular}{|c|c|c|c|c|c|}
\hline $\begin{array}{l}\text { Sample } \\
\text { Code }\end{array}$ & Sample/Activity Description & $\begin{array}{l}\text { Sample } \\
\text { Probe } \\
\text { Line }\end{array}$ & $\begin{array}{l}\text { Sampler Position } \\
\text { During Collection }\end{array}$ & $\begin{array}{l}\text { Sample } \\
\text { Volume }^{2}\end{array}$ & $\begin{array}{c}\text { Sample } \\
\text { Duration or } \\
\text { Flow Rate }\end{array}$ \\
\hline 022 & $\begin{array}{l}\text { Collect particulate filter and tritium } \\
\text { trap sample before pump is started }\end{array}$ & 1 & $\begin{array}{l}\text { 241-AZ-101 } \\
\text { Headspace }\end{array}$ & $1 \mathrm{~L}$ & $\begin{array}{l}50-200 \\
\mathrm{~mL} / \mathrm{min}\end{array}$ \\
\hline 023 & Collect TST & 2 & $\begin{array}{l}\text { 241-AZ-101 } \\
\text { Headspace }\end{array}$ & $0.2 \mathrm{~L}$ & $\begin{array}{c}20-100 \\
\mathrm{~mL} / \mathrm{min}\end{array}$ \\
\hline 024 & Collect filtered TST & 3 & $\begin{array}{l}\text { 241-AZ-101 } \\
\text { Headspace }\end{array}$ & $0.2 \mathrm{~L}$ & $\begin{array}{l}20-100 \\
\mathrm{~mL} / \mathrm{min}\end{array}$ \\
\hline 025 & Collect filtered TST & 4 & $\begin{array}{l}\text { 241-AZ-101 } \\
\text { Headspace }\end{array}$ & $0.2 \mathrm{~L}$ & $\begin{array}{l}20-100 \\
\mathrm{~mL} / \mathrm{min}\end{array}$ \\
\hline 026 & Collect filtered TST & 5 & $\begin{array}{l}241-\mathrm{AZ}-101 \\
\text { Headspace }\end{array}$ & $0.2 \mathrm{~L}$ & $\begin{array}{l}20-100 \\
\mathrm{~mL} / \mathrm{min}\end{array}$ \\
\hline 027 & Collect STT & 6 & $\begin{array}{l}\text { 241-AZ-101 } \\
\text { Headspace }\end{array}$ & $4 \mathrm{~L}$ & $\begin{array}{l}300-500 \\
\mathrm{~mL} / \mathrm{min}\end{array}$ \\
\hline 028 & Collect STT & 7 & $\begin{array}{l}\text { 241-AZ-101 } \\
\text { Headspace }\end{array}$ & $8 \mathrm{~L}$ & $\begin{array}{l}300-500 \\
\mathrm{~mL} / \mathrm{min}\end{array}$ \\
\hline 029 & Collect filtered STT & 8 & $\begin{array}{l}\text { 241-AZ-101 } \\
\text { Headspace }\end{array}$ & $8 \mathrm{~L}$ & $\begin{array}{l}300-500 \\
\mathrm{~mL} / \mathrm{min}\end{array}$ \\
\hline 030 & Collect PUF trap & 9 & $\begin{array}{l}\text { 241-AZ-101 } \\
\text { Headspace }\end{array}$ & $25 \mathrm{~L}$ & $2.5-5 \mathrm{~L} / \mathrm{min}$ \\
\hline 031 & Collect PUF trap & 10 & $\begin{array}{l}\text { 241-AZ-101 } \\
\text { Headspace }\end{array}$ & $25 \mathrm{~L}$ & $2.5-5 \mathrm{~L} / \mathrm{min}$ \\
\hline \multicolumn{6}{|c|}{ Collect sample set \#3 (optional) at direction of Process Engineering. } \\
\hline N/A & $\begin{array}{l}\text { Purge SUMMA }{ }^{\mathrm{TM}} \text { line with tank } \\
\text { vapor. }\end{array}$ & N/A & $\begin{array}{l}\text { 241-AZ-101 } \\
\text { Headspace }\end{array}$ & $5 \mathrm{~L}$ & $1.0-5.0 \mathrm{~L} / \mathrm{min}$ \\
\hline 032 & $\begin{array}{l}\text { Collect SUMMA } \\
\text { canister/particulate filter }\end{array}$ & N/A & $\begin{array}{l}\text { 241-AZ-101 } \\
\text { Headspace }\end{array}$ & $6 \mathrm{~L}$ & $2 \mathrm{~min}$ \\
\hline 033 & $\begin{array}{l}\text { Collect particulate filter and tritium } \\
\text { trap sample }\end{array}$ & 1 & $\begin{array}{l}\text { 241-AZ-101 } \\
\text { Headspace }\end{array}$ & $1 \mathrm{~L}$ & $\begin{array}{c}50-200 \\
\mathrm{~mL} / \mathrm{min}\end{array}$ \\
\hline 034 & Collect TST & 2 & $\begin{array}{l}\text { 241-AZ-101 } \\
\text { Headspace }\end{array}$ & $0.2 \mathrm{~L}$ & $\begin{array}{l}20-100 \\
\mathrm{~mL} / \mathrm{min}\end{array}$ \\
\hline 035 & Collect filtered TST & 3 & $\begin{array}{l}\text { 241-AZ-101 } \\
\text { Headspace }\end{array}$ & $0.2 \mathrm{~L}$ & $\begin{array}{l}20-100 \\
\mathrm{~mL} / \mathrm{min}\end{array}$ \\
\hline 036 & Collect STT & 4 & $\begin{array}{l}\text { 241-AZ-101 } \\
\text { Headspace }\end{array}$ & $8 \mathrm{~L}$ & $\begin{array}{l}300-500 \\
\mathrm{~mL} / \mathrm{min}\end{array}$ \\
\hline 037 & Collect PUF trap & 5 & $\begin{array}{l}\text { 241-AZ-101 } \\
\text { Headspace }\end{array}$ & $25 \mathrm{~L}$ & $2.5-5 \mathrm{~L} / \mathrm{min}$ \\
\hline \multicolumn{6}{|c|}{ Collect sample set \#4 when pump \#1 is first operated in oscillating mode. } \\
\hline $\mathrm{N} / \mathrm{A}$ & $\begin{array}{l}\text { Purge SUMMA }{ }^{\mathrm{TM}} \text { line with tank } \\
\text { vapor. }\end{array}$ & N/A & $\begin{array}{c}241-\mathrm{AZ}-101 \\
\text { Headspace }\end{array}$ & $5 \mathrm{~L}$ & $1.0-5.0 \mathrm{~L} / \mathrm{min}$ \\
\hline 038 & $\begin{array}{l}\text { Collect SUMMA } \\
\text { canister/particulate filter }\end{array}$ & $\mathrm{N} / \mathrm{A}$ & $\begin{array}{l}\text { 241-AZ-101 } \\
\text { Headspace }\end{array}$ & $6 \mathrm{~L}$ & $2 \min$ \\
\hline 039 & $\begin{array}{l}\text { Collect SUMMA }{ }^{\mathrm{TM}} \\
\text { canister/particulate filter }\end{array}$ & N/A & $\begin{array}{l}\text { 241-AZ-101 } \\
\text { Headspace }\end{array}$ & $6 \mathrm{~L}$ & $2 \min$ \\
\hline
\end{tabular}


RPP-5534 Rev. 2

Table 2-3. List of Samples and Activities for 241-AZ-101 Headspace (6 sheets).

\begin{tabular}{|c|c|c|c|c|c|}
\hline $\begin{array}{l}\text { Sample } \\
\text { Code }\end{array}$ & Sample/Activity Description & $\begin{array}{l}\text { Sample } \\
\text { Probe } \\
\text { Line }\end{array}$ & $\begin{array}{l}\text { Sampler Position } \\
\text { During Collection }\end{array}$ & $\begin{array}{l}\text { Sample } \\
\text { Volume }^{2}\end{array}$ & $\begin{array}{c}\text { Sample } \\
\text { Duration or } \\
\text { Flow Rate }\end{array}$ \\
\hline 040 & $\begin{array}{l}\text { Collect SUMMA } \\
\text { canister/particulate filter }\end{array}$ & $\mathrm{N} / \mathrm{A}$ & $\begin{array}{l}\text { 241-AZ-101 } \\
\text { Headspace }\end{array}$ & $6 \mathrm{~L}$ & $2 \min$ \\
\hline 041 & $\begin{array}{l}\text { Collect particulate filter and tritium } \\
\text { trap sample. }\end{array}$ & 1 & $\begin{array}{l}\text { 241-AZ-101 } \\
\text { Headspace }\end{array}$ & $1 \mathrm{~L}$ & $\begin{array}{l}50-200 \\
\mathrm{~mL} / \mathrm{min}\end{array}$ \\
\hline 042 & Collect TST & 2 & $\begin{array}{l}\text { 241-AZ-101 } \\
\text { Headspace }\end{array}$ & $0.2 \mathrm{~L}$ & $\begin{array}{l}20-100 \\
\mathrm{~mL} / \mathrm{min}\end{array}$ \\
\hline 043 & Collect filtered TST & 3 & $\begin{array}{l}\text { 241-AZ-101 } \\
\text { Headspace }\end{array}$ & $0.2 \mathrm{~L}$ & $\begin{array}{l}20-100 \\
\mathrm{~mL} / \mathrm{min}\end{array}$ \\
\hline 044 & Collect filtered TST & 4 & $\begin{array}{l}\text { 241-AZ-101 } \\
\text { Headspace }\end{array}$ & $0.2 \mathrm{~L}$ & $\begin{array}{l}20-100 \\
\mathrm{~mL} / \mathrm{min}\end{array}$ \\
\hline 045 & Collect filtered TST & 5 & $\begin{array}{l}\text { 241-AZ-101 } \\
\text { Headspace }\end{array}$ & $0.2 \mathrm{~L}$ & $\begin{array}{l}20-100 \\
\mathrm{~mL} / \mathrm{min}\end{array}$ \\
\hline 046 & Collect STT & 6 & $\begin{array}{l}\text { 241-AZ-101 } \\
\text { Headspace }\end{array}$ & $4 \mathrm{~L}$ & $\begin{array}{l}300-500 \\
\mathrm{~mL} / \mathrm{min}\end{array}$ \\
\hline 047 & Collect STT & 7 & $\begin{array}{l}\text { 241-AZ-101 } \\
\text { Headspace }\end{array}$ & $8 \mathrm{~L}$ & $\begin{array}{l}300-500 \\
\mathrm{~mL} / \mathrm{min}\end{array}$ \\
\hline 048 & Collect filtered STT & 8 & $\begin{array}{l}\text { 241-AZ-101 } \\
\text { Headspace }\end{array}$ & $8 \mathrm{~L}$ & $\begin{array}{l}300-500 \\
\mathrm{~mL} / \mathrm{min}\end{array}$ \\
\hline 049 & Collect PUF trap & 9 & $\begin{array}{l}\text { 241-AZ-101 } \\
\text { Headspace }\end{array}$ & $25 \mathrm{~L}$ & $2.5-5 \mathrm{~L} / \mathrm{min}$ \\
\hline 050 & Collect PUF trap & 10 & $\begin{array}{l}241-\mathrm{AZ}-101 \\
\text { Headspace }\end{array}$ & $25 \mathrm{~L}$ & $2.5-5 \mathrm{~L} / \mathrm{min}$ \\
\hline \multicolumn{6}{|c|}{ Collect sample set \#5 (optional) at direction of Process Engineering. } \\
\hline $\mathrm{N} / \mathrm{A}$ & $\begin{array}{l}\text { Purge SUMMA }{ }^{\mathrm{TM}} \text { line with tank } \\
\text { vapor. }\end{array}$ & N/A & $\begin{array}{l}241-A Z-101 \\
\text { Headspace }\end{array}$ & $5 \mathrm{~L}$ & $1.0-5.0 \mathrm{~L} / \mathrm{min}$ \\
\hline 051 & $\begin{array}{l}\text { Collect SUMMA } \\
\text { canister/particulate filter }\end{array}$ & $\mathrm{N} / \mathrm{A}$ & $\begin{array}{l}\text { 241-AZ-101 } \\
\text { Headspace }\end{array}$ & $6 \mathrm{~L}$ & $2 \min$ \\
\hline 052 & $\begin{array}{l}\text { Collect particulate filter and tritium } \\
\text { trap sample. }\end{array}$ & 1 & $\begin{array}{c}\text { 241-AZ-101 } \\
\text { Headspace }\end{array}$ & $1 \mathrm{~L}$ & $\begin{array}{l}50-200 \\
\mathrm{~mL} / \mathrm{min}\end{array}$ \\
\hline 053 & Collect TST & 2 & $\begin{array}{l}\text { 241-AZ-101 } \\
\text { Headspace }\end{array}$ & $0.2 \mathrm{~L}$ & $\begin{array}{l}20-100 \\
\mathrm{~mL} / \mathrm{min}\end{array}$ \\
\hline 054 & Collect filtered TST & 3 & $\begin{array}{l}241-\mathrm{AZ}-101 \\
\text { Headspace }\end{array}$ & $0.2 \mathrm{~L}$ & $\begin{array}{l}20-100 \\
\mathrm{~mL} / \mathrm{min}\end{array}$ \\
\hline 055 & Collect STT & 4 & $\begin{array}{l}\text { 241-AZ-101 } \\
\text { Headspace }\end{array}$ & $8 \mathrm{~L}$ & $\begin{array}{l}300-500 \\
\mathrm{~mL} / \mathrm{min}\end{array}$ \\
\hline 056 & Collect PUF trap & 5 & $\begin{array}{l}\text { 241-AZ-101 } \\
\text { Headspace }\end{array}$ & $25 \mathrm{~L}$ & $2.5-5 \mathrm{~L} / \mathrm{min}$ \\
\hline \multicolumn{6}{|c|}{ Collect sample set \#6 (optional) at direction of Process Engineering. } \\
\hline $\mathrm{N} / \mathrm{A}$ & $\begin{array}{l}\text { Purge SUMMA }{ }^{\mathrm{TM}} \text { line with tank } \\
\text { vapor. }\end{array}$ & $\mathrm{N} / \mathrm{A}$ & $\begin{array}{l}\text { 241-AZ-101 } \\
\text { Headspace }\end{array}$ & $5 \mathrm{~L}$ & $1.0-5.0 \mathrm{~L} / \mathrm{min}$ \\
\hline 057 & $\begin{array}{l}\text { Collect SUMMA } \\
\text { canister/particulate filter }\end{array}$ & N/A & $\begin{array}{l}\text { 241-AZ-101 } \\
\text { Headspace }\end{array}$ & $6 \mathrm{~L}$ & $2 \min$ \\
\hline
\end{tabular}


RPP-5534 Rev. 2

Table 2-3. List of Samples and Activities for 241-AZ-101 Headspace (6 sheets).

\begin{tabular}{|c|l|c|c|c|c|}
\hline $\begin{array}{c}\text { Sample } \\
\text { Code }\end{array}$ & \multicolumn{1}{|c|}{ Sample/Activity Description } & $\begin{array}{c}\text { Sample } \\
\text { Probe } \\
\text { Line }\end{array}$ & $\begin{array}{c}\text { Sampler Position } \\
\text { During Collection }\end{array}$ & $\begin{array}{c}\text { Sample } \\
\text { Volume }^{2}\end{array}$ & $\begin{array}{c}\text { Sample } \\
\text { Duration or } \\
\text { Flow Rate }^{1}\end{array}$ \\
\hline 058 & $\begin{array}{l}\text { Collect particulate filter and tritium } \\
\text { trap sample. }\end{array}$ & 1 & $\begin{array}{c}241-\mathrm{AZ}-101 \\
\text { Headspace }\end{array}$ & $1 \mathrm{~L}$ & $\begin{array}{c}50-200 \\
\mathrm{~mL} / \mathrm{min}\end{array}$ \\
\hline 059 & Collect TST & 2 & $\begin{array}{c}241-\mathrm{AZ}-101 \\
\text { Headspace }\end{array}$ & $0.2 \mathrm{~L}$ & $\begin{array}{c}20-100 \\
\mathrm{~mL} / \mathrm{min}\end{array}$ \\
\hline 060 & Collect filtered TST & 3 & $\begin{array}{c}241-\mathrm{AZ}-101 \\
\text { Headspace }\end{array}$ & $0.2 \mathrm{~L}$ & $\begin{array}{c}20-100 \\
\mathrm{~mL} / \mathrm{min}\end{array}$ \\
\hline 061 & Collect STT & 4 & $\begin{array}{c}241-\mathrm{AZ}-101 \\
\text { Headspace }\end{array}$ & $8 \mathrm{~L}$ & $\begin{array}{c}300-500 \\
\mathrm{~mL} / \mathrm{min}\end{array}$ \\
\hline 062 & Collect PUF trap & 5 & $\begin{array}{c}241-\mathrm{AZ}-101 \\
\text { Headspace }\end{array}$ & $25 \mathrm{~L}$ & $2.5-5 \mathrm{~L} / \mathrm{min}$ \\
\hline 063 & Collect PUF trap & 6 & $\begin{array}{c}241-\mathrm{AZ}-101 \\
\text { Headspace }\end{array}$ & $25 \mathrm{~L}$ & $2.5-5 \mathrm{~L} / \mathrm{min}$ \\
\hline
\end{tabular}

Collect sample set \#7 when both mixer pumps are oscillating and operating at maximum speed (1200 rpm).

\begin{tabular}{|c|l|c|c|c|c|}
\hline N/A & $\begin{array}{l}\text { Purge SUMMA TM line with tank } \\
\text { vapor. }\end{array}$ & N/A & $\begin{array}{c}241-\mathrm{AZ}-101 \\
\text { Headspace }\end{array}$ & $5 \mathrm{~L}$ & $5.0 \mathrm{~L} / \mathrm{min}$ \\
\hline 064 & $\begin{array}{l}\text { Collect SUMMA TM } \\
\text { canister/particulate filter }\end{array}$ & N/A & $\begin{array}{c}241-\mathrm{AZ}-101 \\
\text { Headspace }\end{array}$ & $6 \mathrm{~L}$ & $2 \mathrm{~min}$ \\
\hline 065 & $\begin{array}{l}\text { Collect SUMMA TM } \\
\text { canister/particulate filter }\end{array}$ & $\begin{array}{c}241-\mathrm{AZ}-101 \\
\text { Headspace }\end{array}$ & $6 \mathrm{~L}$ & $2 \mathrm{~min}$ \\
\hline 066 & $\begin{array}{l}\text { Collect SUMMA TM } \\
\text { canister/particulate filter }\end{array}$ & N/A & $\begin{array}{c}241-\mathrm{AZ}-101 \\
\text { Headspace }\end{array}$ & $6 \mathrm{~L}$ & $2 \mathrm{~min}$ \\
\hline 067 & $\begin{array}{l}\text { Collect particulate filter and tritium } \\
\text { trap sample }\end{array}$ & 1 & $\begin{array}{c}241-\mathrm{AZ}-101 \\
\text { Headspace }\end{array}$ & $1 \mathrm{~L}$ & $\begin{array}{c}50-200 \\
\mathrm{~mL} / \mathrm{min}\end{array}$ \\
\hline 068 & Collect TST & 2 & $\begin{array}{c}241-\mathrm{AZ}-101 \\
\text { Headspace }\end{array}$ & $0.2 \mathrm{~L}$ & $\begin{array}{c}20-100 \\
\mathrm{~mL} / \mathrm{min}\end{array}$ \\
\hline 069 & Collect filtered TST & 3 & $\begin{array}{c}241-\mathrm{AZ}-101 \\
\text { Headspace }\end{array}$ & $0.2 \mathrm{~L}$ & $\begin{array}{c}20-100 \\
\mathrm{~mL} / \mathrm{min}\end{array}$ \\
\hline 070 & Collect filtered TST & 4 & $\begin{array}{c}241-\mathrm{AZ}-101 \\
\text { Headspace }\end{array}$ & $0.2 \mathrm{~L}$ & $\begin{array}{c}20-100 \\
\mathrm{~mL} / \mathrm{min}\end{array}$ \\
\hline 071 & Collect filtered TST & 5 & $\begin{array}{c}241-\mathrm{AZ}-101 \\
\text { Headspace }\end{array}$ & $0.2 \mathrm{~L}$ & $\begin{array}{c}20-100 \\
\mathrm{~mL} / \mathrm{min}\end{array}$ \\
\hline 072 & Collect STT & 6 & $\begin{array}{c}241-\mathrm{AZ}-101 \\
\text { Headspace }\end{array}$ & $4 \mathrm{~L}$ & $\begin{array}{c}300-500 \\
\mathrm{~mL} / \mathrm{min}\end{array}$ \\
\hline 073 & Collect STT & 7 & $\begin{array}{c}241-\mathrm{AZZ}-101 \\
\text { Headspace }\end{array}$ & $8 \mathrm{~L}$ & $\begin{array}{l}300-500 \\
\mathrm{~mL} / \mathrm{min}\end{array}$ \\
\hline 074 & Collect filtered STT & $\begin{array}{c}241-\mathrm{AZ}-101 \\
\text { Headspace }\end{array}$ & $8 \mathrm{~L}$ & $\begin{array}{l}300-500 \\
\mathrm{~mL} / \mathrm{min}\end{array}$ \\
\hline 075 & Collect PUF trap & $\begin{array}{c}241-\mathrm{AZ}-101 \\
\text { Headspace }\end{array}$ & $25 \mathrm{~L}$ & $2.5-5 \mathrm{~L} / \mathrm{min}$ \\
\hline 076 & Collect PUF trap & 10 & $25 \mathrm{~L}$ & $2.5-5 \mathrm{~L} / \mathrm{min}$ \\
\hline
\end{tabular}


RPP-5534 Rev. 2

Table 2-3. List of Samples and Activities for 241-AZ-101 Headspace (6 sheets).

\begin{tabular}{|c|c|c|c|c|c|}
\hline $\begin{array}{l}\text { Sample } \\
\text { Code }\end{array}$ & Sample/Activity Description & $\begin{array}{c}\text { Sample } \\
\text { Probe } \\
\text { Line }\end{array}$ & $\begin{array}{l}\text { Sampler Position } \\
\text { During Collection }\end{array}$ & $\begin{array}{l}\text { Sample } \\
\text { Volume }^{2}\end{array}$ & $\begin{array}{c}\text { Sample } \\
\text { Duration or } \\
\text { Flow Rate }^{1}\end{array}$ \\
\hline \multicolumn{6}{|c|}{ Collect sample set $\# 8$ (optional) at direction of Process Engineering. } \\
\hline N/A & $\begin{array}{l}\text { Purge SUMMA }{ }^{T M} \text { line with tank } \\
\text { vapor. }\end{array}$ & N/A & $\begin{array}{l}\text { 241-AZ-101 } \\
\text { Headspace }\end{array}$ & $5 \mathrm{~L}$ & $1.0-5.0 \mathrm{~L} / \mathrm{min}$ \\
\hline 077 & $\begin{array}{l}\text { Collect SUMMA } \\
\text { canister/particulate filter }\end{array}$ & N/A & $\begin{array}{l}\text { 241-AZ-101 } \\
\text { Headspace }\end{array}$ & $6 \mathrm{~L}$ & $2 \min$ \\
\hline 078 & $\begin{array}{l}\text { Collect particulate filter and tritium } \\
\text { trap sample }\end{array}$ & 1 & $\begin{array}{l}\text { 241-AZ-101 } \\
\text { Headspace }\end{array}$ & $1 \mathrm{~L}$ & $\begin{array}{l}50-200 \\
\mathrm{~mL} / \mathrm{min}\end{array}$ \\
\hline 079 & Collect TST & 2 & $\begin{array}{l}\text { 241-AZ-101 } \\
\text { Headspace }\end{array}$ & $0.2 \mathrm{~L}$ & $\begin{array}{l}20-100 \\
\mathrm{~mL} / \mathrm{min}\end{array}$ \\
\hline 080 & Collect filtered TST & 3 & $\begin{array}{l}\text { 241-AZ-101 } \\
\text { Headspace }\end{array}$ & $0.2 \mathrm{~L}$ & $\begin{array}{l}20-100 \\
\mathrm{~mL} / \mathrm{min}\end{array}$ \\
\hline 081 & Collect STT & 4 & $\begin{array}{l}\text { 241-AZ-101 } \\
\text { Headspace }\end{array}$ & $8 \mathrm{~L}$ & $\begin{array}{l}300-500 \\
\mathrm{~mL} / \mathrm{min}\end{array}$ \\
\hline 082 & Collect PUF trap & 5 & $\begin{array}{l}241-\mathrm{AZ}-101 \\
\text { Headspace }\end{array}$ & $25 \mathrm{~L}$ & $2.5-5 \mathrm{~L} / \mathrm{min}$ \\
\hline \multicolumn{6}{|c|}{ Blanks } \\
\hline 083 & $\begin{array}{l}\text { Store TST Trip Blank for ambient } \\
\text { samples }\end{array}$ & N/A & --- & --- & --- \\
\hline 084 & Store TST Trip Blank for event \#1 & $\mathrm{N} / \mathrm{A}$ & -- & -- & --- \\
\hline 085 & Store TST Trip Blank for event \#2 & $\mathrm{N} / \mathrm{A}$ & $\cdots$ & --- & $\ddot{---}$ \\
\hline 086 & Store TST Trip Blank for event $\# 3$ & N/A & --- & $\overrightarrow{--}$ & $\cdots$ \\
\hline 087 & Store TST Trip Blank for event \#4 & $\mathrm{N} / \mathrm{A}$ & --- & $\cdots$ & -+ \\
\hline 088 & Store TST Trip Blank for event \#5 & $\mathrm{N} / \mathrm{A}$ & -- & 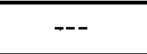 & $\ldots$ \\
\hline 089 & Store TST Trip Blank for event $\# 6$ & $\mathrm{~N} / \mathrm{A}$ & $\cdots$ & --- & -- \\
\hline 090 & Store TST Trip Blank for event \#7 & N/A & --- & $-\cdots$ & $\cdots$ \\
\hline 091 & Store STT Trip Blank for event $\# 2$ & N/A & $\overline{---}$ & --- & -- \\
\hline 092 & Store PUF Trip Blank for event $\# 2$ & N/A & $+\cdots$ & $-\infty$ & -- \\
\hline
\end{tabular}

Note:

${ }^{1} \mathrm{CPO}$ and WSCF at the direction of Process Engineering may adjust sample durations and flow rates as necessary to collect the samples.

${ }^{2}$ The actual sample volume collected is to be within plus or minus $10 \%$ as listed

NEVS $=$ non-electrical vapor sampling system 
RPP-5534 Rev. 2

Table 2-4. Vapor Sampling Summary.

\begin{tabular}{|c|c|c|c|c|}
\hline $\begin{array}{c}\text { Sample } \\
\text { Location }\end{array}$ & Sample Tíme & Sample Type & Sample Container & $\begin{array}{c}\text { Number of } \\
\text { Samples }\end{array}$ \\
\hline \multirow[t]{3}{*}{---} & \multirow[t]{3}{*}{ Baseline } & \multirow[t]{3}{*}{ Trip blank } & TST & Up to 8 \\
\hline & & & SST & 1 \\
\hline & & & PUF & 1 \\
\hline \multirow[t]{4}{*}{---} & \multirow[t]{4}{*}{ Baseline } & \multirow[t]{4}{*}{ Ambient } & SUMMA TM & 2 \\
\hline & & & TST & 1 \\
\hline & & & STT & 1 \\
\hline & & & PUF & 1 \\
\hline \multirow{20}{*}{$\begin{array}{l}241-\mathrm{AZ}-101 \\
\text { Headspace }\end{array}$} & \multirow[t]{5}{*}{ Baseline } & \multirow{5}{*}{$\begin{array}{l}\text { Headspace } \\
\text { Set \#1 }\end{array}$} & SUMMA $^{\mathrm{TM}}$ & 3 \\
\hline & & & TST & 4 \\
\hline & & & STT & 3 \\
\hline & & & PUF & 2 \\
\hline & & & Particulate filter assembly & 1 \\
\hline & \multirow{5}{*}{$\begin{array}{l}\text { During initial } \\
\text { pump operation } \\
\text { of Pump } \# 1\end{array}$} & \multirow{5}{*}{$\begin{array}{l}\text { Headspace } \\
\text { Set \#2 and \#3 }\end{array}$} & SUMMA $^{\mathrm{TM}}$ & Up to 4 \\
\hline & & & TST & Up to 6 \\
\hline & & & STT & Up to 4 \\
\hline & & & PUF & Up to 3 \\
\hline & & & Particulate filter assembly & Up to 2 \\
\hline & \multirow{5}{*}{$\begin{array}{l}\text { When Pump \#1 } \\
\text { is first operated } \\
\text { in oscillating } \\
\text { mode. }\end{array}$} & \multirow{5}{*}{$\begin{array}{l}\text { Headspace } \\
\text { Set \#4, \#5, and } \\
\# 6\end{array}$} & SUMMA $^{\mathrm{TM}}$ & Up to 5 \\
\hline & & & TST & Up to 8 \\
\hline & & & STT & Up to 5 \\
\hline & & & PUF & Up to 5 \\
\hline & & & Particulate filter assembly & Up to 3 \\
\hline & \multirow{5}{*}{$\begin{array}{l}\text { When both } \\
\text { mixer pumps are } \\
\text { operating at } \\
\text { maximum speed }\end{array}$} & \multirow{5}{*}{$\begin{array}{l}\text { Headspace } \\
\text { Set \#7 and \#8 }\end{array}$} & SUMMA ${ }^{\mathrm{TM}}$ & Up to 4 \\
\hline & & & TST & Up to 6 \\
\hline & & & STT & Up to 4 \\
\hline & & & PUF & Up to 3 \\
\hline & & & Particulate filter assembly & Up to 2 \\
\hline
\end{tabular}

Notes:

Baseline $=$ prior to pump startup

Each particulate filter assembly contains two particulate filters mounted in series. 


\subsection{RADIATION SCREENING}

Surveys using particulate filter samples and tritium traps from the 241-AZ-101 headspace will be performed during the test on selected samples. Particulate filters will be located upstream of the SUMMA $^{\mathrm{TM}}$ canisters, three out of four of the TST per set and one STT per set during sample collection. The particulate filters will be used to allow the samples to obtain a radiological release and ensure that the samples meet the WSCF laboratory acceptance criteria. The particulate filter samples will be collected by CPO and sent to 222-S Laboratory for analysis of both the upstream and downstream particulate filters in each particulate filter assembly. Analytical procedures are specified in Section 3.0. The results from the radiation screening shall be submitted to CPO, WSCF, the 222-S Project Coordinator, and the Process Engineering point of contact for vapor sampling for evaluation. 
RPP-5534 Rev. 2

\subsection{LABORATORY ANALYSIS REQUIREMENTS}

The responsibilities of the analytical laboratories are given in this section. Additional quality control and deliverable requirements are given in Sections 4.0 and 7.0. All samples shall be stored at a nominal 4 degrees Celsius prior to extraction/preparation.

Vapor samples shall be analyzed by WSCF in accordance with Table 3-1. Samples that do not meet the WSCF acceptance criteria will be analyzed at the 222-S Laboratory or another on-site laboratory (Pacific Northwest National Laboratory [PNNL]). Sorbent tube trains shall be analyzed for ammonia and oxides of nitrogen (nitric oxide and nitrogen dioxide). SUMMA ${ }^{\mathrm{TM}}$ canisters shall be analyzed for total non-methane hydrocarbons, hydrogen, nitrous oxide, methane, carbon monoxide, carbon dioxide, and selected organic analytes. TSTs shall be analyzed for selected organic analytes. PUF samples shall be analyzed for TBP and DBP.

Required analytes are those listed in Tables 3-2. Quantitation limit goals for the Class A and B toxic air pollutants in these tables are documented in Mulkey (1999). Because of the large number of analytes of interest, quantification will be conducted only on the analytes in Table 3-2. Tentative identification will be conducted for all other organics whose chromatographs indicate they are at a concentration of $1 \mathrm{ppmv}$ or above except for polychlorinated biphenyls which shall be reported at any level. All major constituents in the sample should be identified.

It is possible that not all samples will be analyzed. It is expected that, at a minimum, the laboratory will be directed to analyze all trip blanks, field blanks, baseline samples, and at least four sets of samples from tank 241-AZ-101 headspace. Prior to the receipt of the samples at the laboratory, the Process Engineering point of contact for vapor sampling shall provide WSCF with a list identifying the samples not to be analyzed if necessary.

Particulate filter samples shall be analyzed at the WSCF or 222-S Laboratory as discussed in Section 2.3 and in accordance with Table 3-1.

If any requested analyses cannot be performed, the Process Engineering point of contact for vapor sampling shall be notified. 
RPP-5534 Rev. 2

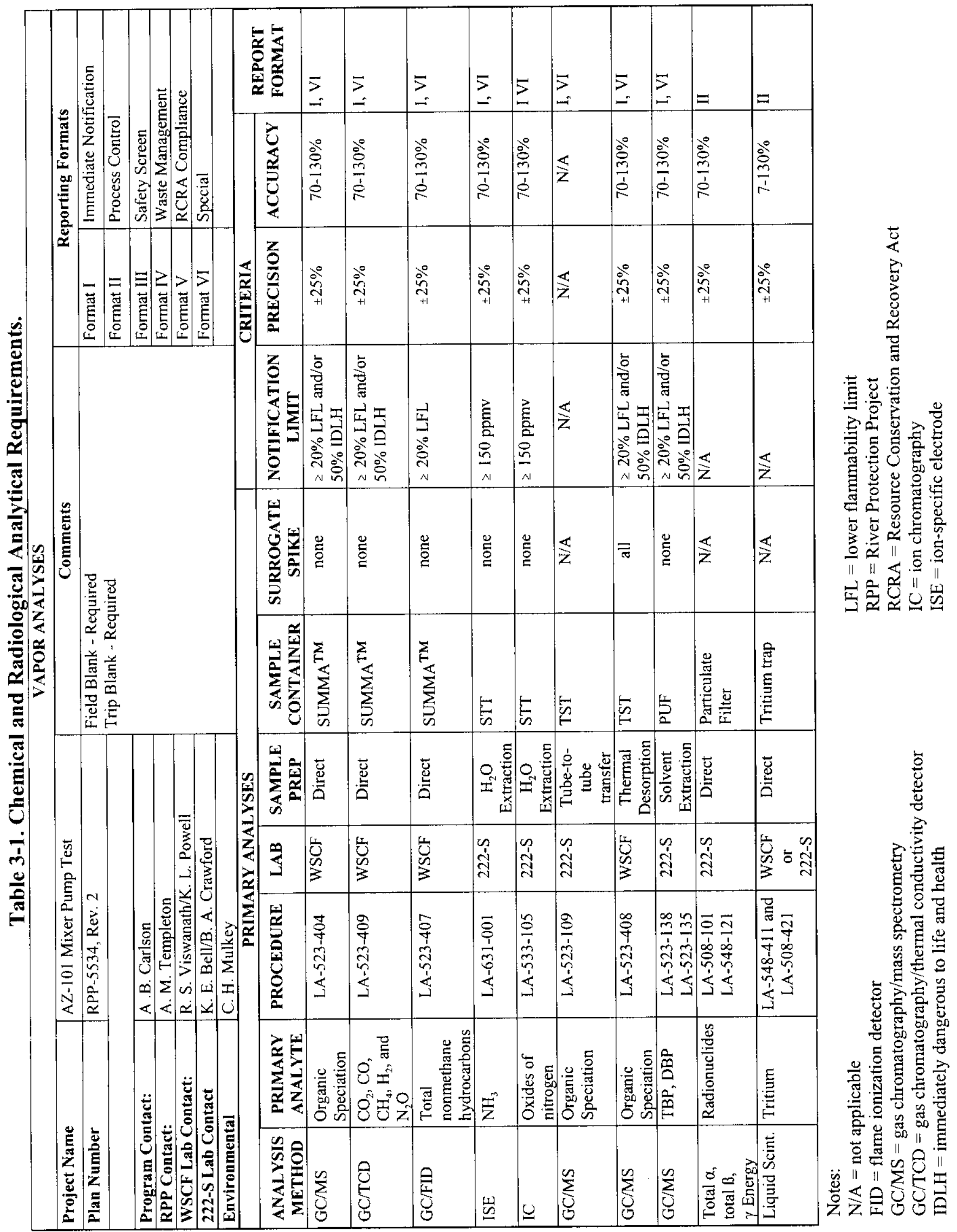


RPP-5534 Rev. 2

Table 3-2. Vapor Analytes and Quantitation Limits. (4 sheets)

\begin{tabular}{|c|c|c|c|}
\hline Analyte & $\begin{array}{c}\text { CAS } \\
\text { Number }\end{array}$ & $\begin{array}{l}\text { VPRQL } \\
\left(\mathrm{mg} / \mathrm{m}^{\mathbf{3}}\right)\end{array}$ & $\begin{array}{c}\text { VPRQL } \\
\text { (ppbv) }\end{array}$ \\
\hline \multicolumn{4}{|l|}{ VOCs in TST Samples } \\
\hline Acetone & $67-64-1$ & 0.15 & 63 \\
\hline Acetonitrile & $75-05-8$ & 0.15 & 89 \\
\hline Benzene & $71-43-2$ & 0.15 & 47 \\
\hline 1-Butanol & $71-36-3$ & 0.15 & 49 \\
\hline Dodecane & $112-40-3$ & 0.15 & 22 \\
\hline n-Hexane & $110-54-3$ & 0.15 & 43 \\
\hline Propanenitrile & $107-12-0$ & 0.15 & 67 \\
\hline Tridecane & $629-50-5$ & 0.15 & 20 \\
\hline Dichloromethane (methylene chloride) & $75-09-2$ & 0.15 & 43 \\
\hline Butanenitrile & $109-74-0$ & 0.15 & 53 \\
\hline 2-Pentanone & $107-87-9$ & 0.15 & 43 \\
\hline n-Heptane & $142-82-5$ & 0.15 & 37 \\
\hline Methyl benzene (toluene) & $108-88-3$ & 0.15 & 40 \\
\hline Pentanenitrile & $110-59-8$ & 0.15 & 44 \\
\hline 2-Hexanone (methyl butyl ketone) & $591-78-6$ & 0.15 & 37 \\
\hline Octane & $111-65-9$ & 0.15 & 32 \\
\hline Hexanenitrile & $628-73-9$ & 0.15 & 38 \\
\hline 2-Heptanone & $110-43-0$ & 0.15 & 32 \\
\hline n-Nonane & $111-84-2$ & 0.15 & 29 \\
\hline Heptanenitrile & $629-08-3$ & 0.15 & 33 \\
\hline 2-Octanone & $111-13-7$ & 0.15 & 29 \\
\hline Decane & $124-18-5$ & 0.15 & 26 \\
\hline Undecane & $1120-21-4$ & 0.15 & 23 \\
\hline Methanol & $7-56-1$ & 0.15 & 110 \\
\hline Ethanol & $64-17-5$ & 0.15 & 80 \\
\hline 1-Propanol & $71-23-8$ & 0.15 & 61 \\
\hline Tetrahydrofuran & 109-99-9 & 0.15 & 51 \\
\hline 1,2, 4-Trimethylbenzene & $95-63-6$ & 0.15 & 30 \\
\hline o-Xylene & $95-47-6$ & 0.15 & 34 \\
\hline Carbon tetrachloride & $56-23-5$ & 0.15 & 24 \\
\hline Chloroform & $67-66-3$ & 0.15 & 31 \\
\hline
\end{tabular}


RPP-5534 Rev. 2

Table 3-2. Vapor Analytes and Quantitation Limits. (4 sheets)

\begin{tabular}{|c|c|c|c|}
\hline Analyte & $\begin{array}{c}\text { CAS } \\
\text { Number }\end{array}$ & $\begin{array}{l}\text { VPRQL } \\
\left(\mathrm{mg} / \mathrm{m}^{3}\right)\end{array}$ & $\begin{array}{c}\text { VPRQL } \\
\text { (ppbv) }\end{array}$ \\
\hline 1,2 dichloroethane & $107-06-2$ & 0.15 & TBD \\
\hline 1,1 dichloroethylene & $75-35-4$ & 0.15 & TBD \\
\hline 2,4 dinitrotoluene & $121-14-2$ & 0.15 & TBD \\
\hline Hexachlorobutadiene & $87-68-3$ & 0.15 & TBD \\
\hline Hexachloroethane & $67-72-1$ & 0.15 & TBD \\
\hline Methyl ethyl ketone & $78-93-3$ & 0.15 & TBD \\
\hline Nitrobenzene & $98-95-3$ & 0.15 & TBD \\
\hline Pyridine & $110-86-1$ & 0.15 & TBD \\
\hline Trichloroethylene & $79-01-6$ & 0.15 & TBD \\
\hline $2,4,5$ trichlorophenol & $95-95-4$ & 0.15 & TBD \\
\hline Vinyl chloride & $75-01-4$ & 0.15 & TBD \\
\hline Tetrachloroethylene & $127-18-4$ & 0.15 & 22 \\
\hline \multicolumn{4}{|l|}{ Permanent Gases in SUMMA ${ }^{\mathrm{TM}}$ Samples } \\
\hline Carbon Dioxide & $124-38-9$ & 90 & 50 ppmv \\
\hline Carbon Monoxide & $630-08-0$ & 57 & $50 \mathrm{ppmv}$ \\
\hline Hydrogen & $1333-74-0$ & 4.1 .0 & 50 ppmv \\
\hline Methane & $74-82-8$ & 33 & 50 ppmv \\
\hline Nitrous oxide & $10024-97-2$ & 90 & 50 ppmv \\
\hline \multicolumn{4}{|l|}{ TNMOC in SUMMA ${ }^{\mathrm{TM}}$ Samples } \\
\hline Total non-methane organic compounds & & 0.07 & 150 \\
\hline \multicolumn{4}{|l|}{ VOCs in SUMMA ${ }^{\mathrm{TM}}$ Samples } \\
\hline Acetone & $67-64-1$ & 0.012 & 5.0 \\
\hline Acetonitrile & $75-05-8$ & 0.008 & 5.0 \\
\hline Benzene & $71-43-2$ & 0.016 & 5.0 \\
\hline Butanal & $123-72-8$ & 0.015 & 5.0 \\
\hline 1-Butanol & $71-36-3$ & 0.015 & 5.0 \\
\hline Propanenitrile & $107-12-0$ & 0.011 & 5.0 \\
\hline Chloroethane & $75-00-3$ & 0.013 & 5.0 \\
\hline Trichlorodifluoromethane (Freon 12) & $75-71-8$ & 0.025 & 5.0 \\
\hline Methylbenzene & $108-88-3$ & 0.019 & 5.0 \\
\hline Ethylbenzene & $100-41-4$ & 0.022 & 5.0 \\
\hline 2-Butanone & $78-93-3$ & 0.015 & 5.0 \\
\hline
\end{tabular}


RPP-5534 Rev. 2

Table 3-2. Vapor Analytes and Quantitation Limits. (4 sheets)

\begin{tabular}{|c|c|c|c|}
\hline Analyte & $\begin{array}{c}\text { CAS } \\
\text { Number }\end{array}$ & $\begin{array}{l}\text { VPRQL } \\
\left(\mathrm{mg} / \mathrm{m}^{3}\right)\end{array}$ & $\begin{array}{l}\text { VPRQL } \\
\text { (ppbv) }\end{array}$ \\
\hline n-Heptane & $142-72-5$ & 0.020 & 5.0 \\
\hline Tetrahydrofuran & $109-99-9$ & 0.015 & 5.0 \\
\hline 2-Propanol & $67-63-0$ & 0.012 & 5.0 \\
\hline 1-Propanol & $71-23-8$ & 0.012 & 5.0 \\
\hline Trichlorofluoromethane (Freon 11) & $75-69-4$ & 0.028 & 5.0 \\
\hline o-Xylene & $95-47-6$ & 0.022 & 5.0 \\
\hline m-Xylene & $108-38-3$ & 0.022 & 5.0 \\
\hline p-Xylene & $106-42-3$ & 0.022 & 5.0 \\
\hline 1,2,4-Trimethylbenzene & $95-63-6$ & 0.024 & 5.0 \\
\hline Decane & $124-18-5$ & 0.029 & 5.0 \\
\hline 4-Methyl-2-pentanone & $108-10-1$ & 0.020 & 5.0 \\
\hline Butanenitrile & $109-74-0$ & 0.014 & 5.0 \\
\hline Octane & $111-65-9$ & 0.023 & 5.0 \\
\hline Tetrachloroethylene & $127-18-4$ & 0.034 & 5.0 \\
\hline Chloromethane & $74-87-3$ & 0.010 & 5.0 \\
\hline Dichloromethane (methylene chloride) & $75-09-2$ & 0.017 & 5.0 \\
\hline Chloroform (trichloromethane) & $67-66-3$ & 0.024 & 5.0 \\
\hline $\begin{array}{l}\text { Carbon tetrachloride } \\
\text { (tetrachloromethane) }\end{array}$ & $56-23-5$ & 0.031 & 5.0 \\
\hline Pentane & $109-66-0$ & 0.015 & 5.0 \\
\hline 2-Methylpentane & $107-83-5$ & 0.018 & 5.0 \\
\hline 1 -Hexene & $592-41-6$ & 0.017 & 5.0 \\
\hline n-Butane & $106-97-8$ & 0.012 & 5.0 \\
\hline 3-Methyl hexane & $598-34-4$ & 0.020 & 5.0 \\
\hline 1,4-Diethylene dioxide (dioxin) & $123-91-1$ & 0.018 & 5.0 \\
\hline Chlorobenzene & $108-90-7$ & 0.023 & 5.0 \\
\hline Styrene & $100-42-5$ & 0.021 & 5.0 \\
\hline 3-Heptanone & $106-35-4$ & 0.023 & 5.0 \\
\hline Cyclohexanone & $108-94-1$ & 0.20 & 5.0 \\
\hline Furan & $110-00-9$ & 0.014 & 5.0 \\
\hline $\mathrm{n}$-Nonane & $111-84-2$ & 0.026 & 5.0 \\
\hline Ethanol & $64-17-5$ & 0.009 & 5.0 \\
\hline 2-Pentanone & $107-87-9$ & 0.018 & 5.0 \\
\hline
\end{tabular}


RPP-5534 Rev. 2

Table 3-2. Vapor Analytes and Quantitation Limits. (4 sheets)

\begin{tabular}{|l|c|c|c|}
\hline \multicolumn{1}{|c|}{ Analyte } & $\begin{array}{c}\text { CAS } \\
\text { Number }\end{array}$ & $\begin{array}{c}\text { VPRQL } \\
\left(\mathbf{m g} / \mathbf{m}^{\mathbf{3}}\right)\end{array}$ & $\begin{array}{c}\text { VPRQL } \\
(\mathbf{p p b v})\end{array}$ \\
\hline 2-Hexanone & $591-78-6$ & 0.020 & 5.0 \\
\hline 2-Heptanone & $110-43-0$ & 0.023 & 5.0 \\
\hline 1,2 dichloroethane & $107-06-2$ & 0.15 & TBD \\
\hline 1,1 dichloroethylene & $75-35-4$ & 0.15 & TBD \\
\hline 2,4 dinitrotoluene & $121-14-2$ & 0.15 & TBD \\
\hline Hexachlorobutadiene & $87-68-3$ & 0.15 & TBD \\
\hline Hexachloroethane & $67-72-1$ & 0.15 & TBD \\
\hline Methyl ethyl ketone & $78-93-3$ & 0.15 & TBD \\
\hline Nitrobenzene & $98-95-3$ & 0.15 & TBD \\
\hline Pyridine & $110-86-1$ & 0.15 & TBD \\
\hline Trichloroethylene & $79-01-6$ & 0.15 & TBD \\
\hline 2,4,5 trichlorophenol & $95-95-4$ & 0.15 & TBD \\
\hline Vinyl chloride & $75-01-4$ & 0.15 & TBD \\
\hline 2-Octanone & $111-13-7$ & 0.026 & 5.0 \\
\hline PUF & \multicolumn{3}{|l}{} \\
\hline Dibutyl phosphate & $107-66-4$ & & 100 \\
\hline Tributyl phosphate & $126-73-8$ & & 100 \\
\hline Inorganic Sorbent Tube Train Samples & & \\
\hline Ammonia & $7664-41-7$ & 10 & $14 \mathrm{ppmv}$ \\
\hline Nitrogen dioxide & $10102-44-0$ & 5.0 & $2.7 \mathrm{ppmv}$ \\
\hline Nitrous oxide & $10102-43-9$ & 5.0 & $4.1 \mathrm{ppmv}$ \\
\hline
\end{tabular}

Notes:

$\mathrm{CAS}=$ Chemical Abstracts Service

$\mathrm{PUF}=$ polyurethane foam

VPRQL $=$ Vapor Program Required Quantitation Limit

TNMOC $=$ Total non-methane organic compounds 


\subsection{QUALITY ASSURANCE AND QUALITY CONTROL}

Vapor sampling and analysis shall be performed in accordance with approved quality assurance (QA) plans. These plans are required to meet the Hanford Analytical Services Quality Assurance Requirements Document (HASQARD) (DOE 1998) requirements. Validation of this compliance shall be verified either by a HASQARD assessment stating their quality program satisfactorily meets the appropriate requirements, or the quality program plan and applicable procedures will be submitted and approved prior to work performance on sampling or analytical work. Quality requirements for conducting Characterization Project sampling and analysis are described in Tank Waste Remediation System Characterization Project, Quality Policies (Board 1998) and this sampling and analysis plan. Characterization Project sampling and analysis shall be conducted in conformance with these QA requirements.

Processes, services, activities, and conditions adverse to quality which do not conform to requirements specified in this sampling and analysis plan or references herein shall be controlled to prevent inadvertent use. Nonconforming sampling and analysis processes shall be identified, controlled, reported, and dispositioned as required by CHG (1999).

\subsection{LABORATORY OPERATIONS}

Analytical procedures that are to be used for this sample and analysis plan shall be written and approved prior to the performance of analytical work within the laboratory. Analytical QC requirements are identified in Table 3-1. The laboratory shall also use calibration and calibration check standards appropriate for the analytical instrumentation being used (see DOE [1998] for definitions of QC samples and standards). The criteria presented are goals for demonstrating reliable method performance. It is understood that the laboratory will follow its internal QC system for required actions whenever QC failures occur. If sample QC failures occur or if all analyses cannot be performed (e.g., insufficient sample), analysts shall consult with supervisors/customers to determine the proper action. The laboratory should provide a suggested course of action at that time. All sample QC failures and limitations on the associated data shall be discussed in the narrative of the data report. Proper notification of all data not meeting QC requirements shall be included with the data.

\subsection{SAMPLE COLLECTION}

Sampling shall be performed in accordance with approved procedures and work plans included within the job control system. All data sheets and log entries completed during the performance of sampling shall be copied and included within the job control system package.

Each sample identification number shall have the following format: 
where,

$\mathbf{V}$ indicates a vapor sample,

$\mathbf{W}=$ a letter code identifying the organization that prepared the sample container/sample media $\mathrm{W}=\mathrm{WSCF}$,

$\mathbf{X X X}=$ a three-digit/letter code identifying the sample location

$101=241-\mathrm{AZ}-101$ headspace

$\mathrm{AMB}=$ ambient air samples at 241-AZ tank farm

YYY $=$ a three-digit sample code found in Table 2-3

$\mathbf{Z Z Z Z}=$ a special lab-assigned code.

\subsection{SAMPLE CUSTODY}

Chain-of-custody will be generated at the time of sample head assembly and carefully maintained to assure sample control at all times. Documents (i.e., SAP), procedures, and drawings (including current revision number) applicable to the sample head assembly will be listed on the chain-of-custody. 
RPP-5534 Rev. 2

\subsection{EXCEPTIONS, CLARIFICATIONS, AND ASSUMPTIONS}

\subsection{CLARIFICATIONS}

\section{Trip Blanks and Field Blanks}

Trip Blanks are sampling devices prepared and handled in the same manner as samples, except that they are never opened in the field. Field Blanks are sampling devices prepared and handled in the same manner as the samples, but no tank gases are drawn through them.

\subsection{EXCEPTIONS}

The analytes required for the vapor analyses are found in Mulkey (1999) (Table 8-3 and Table C-1). Only a subset of the analytes listed in Table C-1 will be included for analysis. This subset is the analytes included for the tank 241-C-106 vapor analyses and is shown in Table 3-2. 


\subsection{ORGANIZATION}

The organization and responsibility of key personnel involved with these tank characterization projects are listed in Table 6-1.

Table 6-1. Project Key Personnel

\begin{tabular}{|c|c|c|}
\hline Responsibility & Organization & Individual \\
\hline $\begin{array}{l}\text { Data Development and } \\
\text { Interpretation manager }\end{array}$ & RPP Process Engineering (CHG) & J. G. Field, 376-3753 \\
\hline $\begin{array}{l}\text { Process Engineering point of } \\
\text { contact for AZ-101 vapor } \\
\text { sampling }\end{array}$ & RPP Process Engineering ( $\mathrm{CHG}$ ) & A. M. Templeton, 373-5589 \\
\hline $\begin{array}{l}\text { Waste Retrieval Mixer Pump } \\
\text { test technical contact }\end{array}$ & Retrieval Engineering (NHC) & A. B. Carlson, 376-1642 \\
\hline $\begin{array}{l}\text { Process Engineering mixer } \\
\text { pump operation contact }\end{array}$ & $\begin{array}{l}\text { RPP Process Engineering, } \\
(\mathrm{CHG})\end{array}$ & K. G. Carothers, 373-4556 \\
\hline $\begin{array}{l}\text { Vapor Sampling cognizant } \\
\text { engineer }\end{array}$ & $\begin{array}{l}\text { Characterization Field } \\
\text { Engineering }(\mathrm{CHG})\end{array}$ & D. D. Wanner, 373-3297 \\
\hline Vapor Sampling manager & $\begin{array}{l}\text { Characterization Project } \\
\text { Operations }(\mathrm{CPO})(\mathrm{CHG})\end{array}$ & $\begin{array}{l}\text { J. F. Sickels, 373-0259 } \\
\text { R. J. Praznik, 373-7751 }\end{array}$ \\
\hline $\begin{array}{l}\text { Client Services, Analytical } \\
\text { Services Project }\end{array}$ & Fluor Hanford & K. L. Powell, 372-0939 \\
\hline $\begin{array}{l}\text { Team Lead, Organic } \\
\text { Chemistry, WSCF }\end{array}$ & Fluor Hanford & R. S. Viswanath,373-9223 \\
\hline $\begin{array}{l}\text { Analytical Production, } \\
\text { WSCF Laboratory }\end{array}$ & Fluor Hanford & K. J. Greenough, 373-7194 \\
\hline $\begin{array}{l}\text { Analytical Production, } \\
\text { WSCF Laboratory }\end{array}$ & Fluor Hanford & S. L. Cobb, 373-7118 \\
\hline $\begin{array}{l}\text { Analytical Production, } \\
\text { 222-S Laboratory }\end{array}$ & Fluor Hanford & B. A. Crawford, 373-1972 \\
\hline $\begin{array}{l}\text { 222-S Laboratory Project } \\
\text { Coordinator }\end{array}$ & $\begin{array}{l}\text { Project Assistance Corporation } \\
\text { (PAC) }\end{array}$ & K. E. Bell, 372-2553 \\
\hline $\begin{array}{l}\text { Industrial Hygiene and Safety } \\
\text { points of contact }\end{array}$ & $\begin{array}{l}\text { Tank Farm Facilities Operations, } \\
\text { Field Safety Services (CHG) }\end{array}$ & R. J. Fogg, 373-0630 \\
\hline $\begin{array}{l}\text { Double-Shell Tank Farm } \\
\text { point of contact }\end{array}$ & Tank Farm Operations & $\begin{array}{l}\text { Double-Shell Tank Farm } \\
\text { Operations shift manager, } \\
373-2689\end{array}$ \\
\hline $\begin{array}{l}\text { Environmental point of } \\
\text { contact }\end{array}$ & Environmental Services (CHG) & $\begin{array}{l}\text { C. H. Mulkey, 373-0956 } \\
\text { J. J. Luke, 376-8629 }\end{array}$ \\
\hline
\end{tabular}

CHG $=$ CH2M HILL Hanford Group, Inc. 


\subsection{DELIVERABLES}

Sampling and analytical results shall be reported as Format VI reports. Any analyte exceeding the notification limit prescribed in Table 3-1 shall also be reported as a Format I report. In addition, Format II reports shall be provided by CPO, and WSCF as described in Section 7.2.

\subsection{FORMAT I REPORTING}

Table 3-1 contains the notification limits for specific analytes. Analytes that exceed notification limits shall be reported by the Project Manager or delegate by calling the Double-Shell Tank Farms Operations shift manager as soon as the data are obtained and reviewed by the responsible scientist. This verbal notification must be followed within one hour by electronic notification to the Double-Shell Tank Farms Operations shift manager, the Industrial Hygiene and Safety point of contact, the RPP Process Engineering Data Development and Interpretation manager, and the Process Engineering point of contact for vapor sampling. A further review of the data, including quality control results and additional analyses for verification purposes may be contracted with the performing laboratory by either a revision to this sampling and analysis plan or by a letter.

\subsection{FORMAT II REPORTING}

WSCF shall provide the results of radiological analyses using the standard WSCF analytical laboratory report format. The results shall be faxed to the vapor analysis team at WSCF and the Process Engineering vapor sampling point of contact within 48 hours of receipt of the samples and followed by transmittal of a copy of the results via plant mail.

CPO shall provide information on its sampling activities (copies of sampling data sheets, J-5 forms, chain of custody forms, and other pertinent documentation) to WSCF, the 222-S Project Coordinator, and the Process Engineering vapor sampling point of contact. The information provided shall include: the sample collection sequence and volumes, start and stop times for the collection of each sample, the VOC reading at the start of the collection of each sample, and any anomalous sampling conditions. This information shall be provided within 48 hours of the collection of the last set of samples.

\subsection{FORMAT VI REPORTING}

The Format VI report shall consist of two deliverables, preliminary analytical results, and a final data package.

Preliminary sampling and analytical data shall be delivered within three weeks of the receipt of the samples at the laboratory. The receipt at the laboratory is defined as after radiological screening results are complete and the samples are released to the laboratory for headspace vapor analysis. In regards to any vapor analysis or extraction performed at 222-S laboratory the 
turnaround for preliminary data will be 5 weeks after delivery to the laboratory. The preliminary data shall consist of, at a minimum, data tables reporting sample collection data, particulate filter analysis results, and the results of each analysis performed by the analytical laboratory. The following individuals shall be on distribution for the preliminary results: A. B. Carlson, C. H. Mulkey, K. G. Carothers, G. M. Crummel, A. M. Templeton, G. R. Tardiff, and L. M. Sasaki.

A data package shall be issued as a supporting document within ten weeks of the receipt of the samples at the laboratory. The data package shall contain the elements listed in Table 7-1. The following individuals shall be on distribution for the entire data package: R. A. Bechtold, C. H. Mulkey, G. M. Crummel, A. M. Templeton, and A. E. Young. The following individuals require only the engineering data transmittal: K. G. Carothers, A. B. Carlson, and C. A. Simonen.

In addition to the data package, an electronic version of the analytical results shall be provided to the Tank Vapor Database representative within 4 calendar days from the day that the final data package is issued. The data must be available to the Washington State Department of Ecology within 7 calendar days of release of the data package. The electronic version shall be in the standard electronic format specified in Bobrowski and Simonen (1999).

Table 7-1. Data Package Required Elements (2 sheets). ${ }^{1}$

\begin{tabular}{|l|}
\hline \multicolumn{1}{|c|}{ Prefatory Elements } \\
\hline Executive summary \\
\hline Table of contents \\
\hline List of abbreviations and acronyms \\
\hline Quality assurance data package review results \\
\hline Non-conformance reports \\
\hline \\
\hline Sampling case narrative \\
\hline Sample summary and event chronology \\
\hline Sampling procedures table \\
\hline Sampling logbook table \\
\hline Field data \\
\hline Radiation screening results \\
\hline Chain of custody forms \\
\hline
\end{tabular}


RPP-5534 Rev. 2

Table 7-1. Data Package Required Elements (2 sheets). ${ }^{1}$

\begin{tabular}{|l|}
\hline \multicolumn{1}{|c|}{ Analysis Elements } \\
\hline Analytical case narrative \\
\hline Analytical procedures table \\
\hline Data qualifier flag translation table \\
\hline Target analytes concentration table \\
\hline Tentatively identified compound concentration table \\
\hline Laboratory blank summary \\
\hline Field blank summary \\
\hline Trip blank summary \\
\hline Mass spectrometer instrument tune report \\
\hline Target analyte initial calibration table \\
\hline Internal standards area counts table \\
\hline Laboratory control sample results table \\
\hline Surrogate compounds results table \\
\hline Quantitation reports \\
\hline Chromatograms \\
\hline Mass spectra of reported tentatively identified compounds \\
\hline
\end{tabular}

Note:

${ }^{1}$ Include all elements as applicable. 


\subsection{CHANGE CONTROL}

Under certain circumstances, it may become necessary for the performing laboratory to make decisions concerning a sample without review of the data by the customer or the Characterization Project. All significant changes shall be documented by RPP Process Engineering via an engineering change notice to this sampling and analysis plan or by a letter. All changes shall also be clearly documented in the final data report. Insignificant changes may be made by placing a notation in the permanent record (i.e., note change in logbook or memorandum to file). Significance is determined by the Process Engineering point of contact for vapor sampling. 


\subsection{REFERENCES}

Banning, D. L., 1999, Tank 241-AZ-101 Mixer Pump Test Data Quality Objective, RPP-5498, Rev. 0, Lockheed Martin Hanford Corp., Richland, Washington.

Board, D. C., 1998, Tank Waste Remediation System, Characterization Project, Quality Policies, HNF-SD-WM-QAPP-025, Rev. 4, Lockheed Martin Hanford Corp. for Fluor Daniel Hanford, Inc., Richland, Washington.

Bobrowski, S. F., and C. A. Simonen, 1999, Standard Electronic Format Specification for Tank Vapor Data MSEXCEL Spreadsheets: Version 1.0, HNF-3815, Rev. 0, prepared by Pacific Northwest National Laboratory for Lockheed Martin Hanford Corp, Richland, Washington.

DOE, 1998, Hanford Analytical Services Quality Assurance Requirements Document, DOE/RL-96-68, Rev. 2, U.S. Department of Energy, Richland Operations Office, Richland, Washington.

Fogg, R. J., 2000, 241-AZ-101 IH Sampling/Monitoring Strategy - Rev/ 1, (internal memorandum 7B100-RJF-00-002, to J. L. Homan, January 24), CH2M HILL Hanford Group, Inc., Richland, Washington.

Hodgson, K. M., 1995, Tank Characterization Report for Double-Shell Tank 241-AZ-101, WHC-SD-WM-ER-410, Westinghouse Hanford Company, Richland, Washington.

CHG, 1999, Nonconforming Item Reporting and Control, RPP-PRO-298, Rev. 0, CH2M HILL Hanford Group, Inc., Richland, Washington.

Mulkey, C. H., 1999, Data Quality Objectives for Regulatory Requirements for Hazardous and Radioactive Air Emissions Sampling and Analysis, WHC-SD-WM-DQO-021, Rev. 1, Lockheed Martin Hanford Corp., Richland, Washington.

Reich, F. R., 2000, Operations Test Plan for the Type 4 In-Situ Vapor Sampler (ISVS) System, RPP-5659, Rev. 0, Prepared by COGEMA Engineering Corporation for CH2M HILL Hanford Group, Inc., Richland, Washington. 
RPP-5534 Rev. 2

APPENDIX A

AZ-101 CORE PROFILE

A-1 


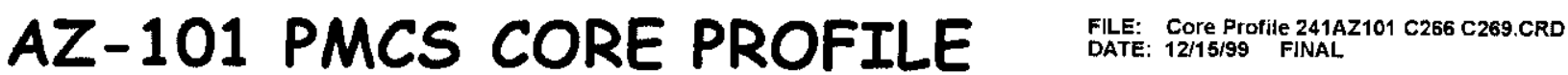

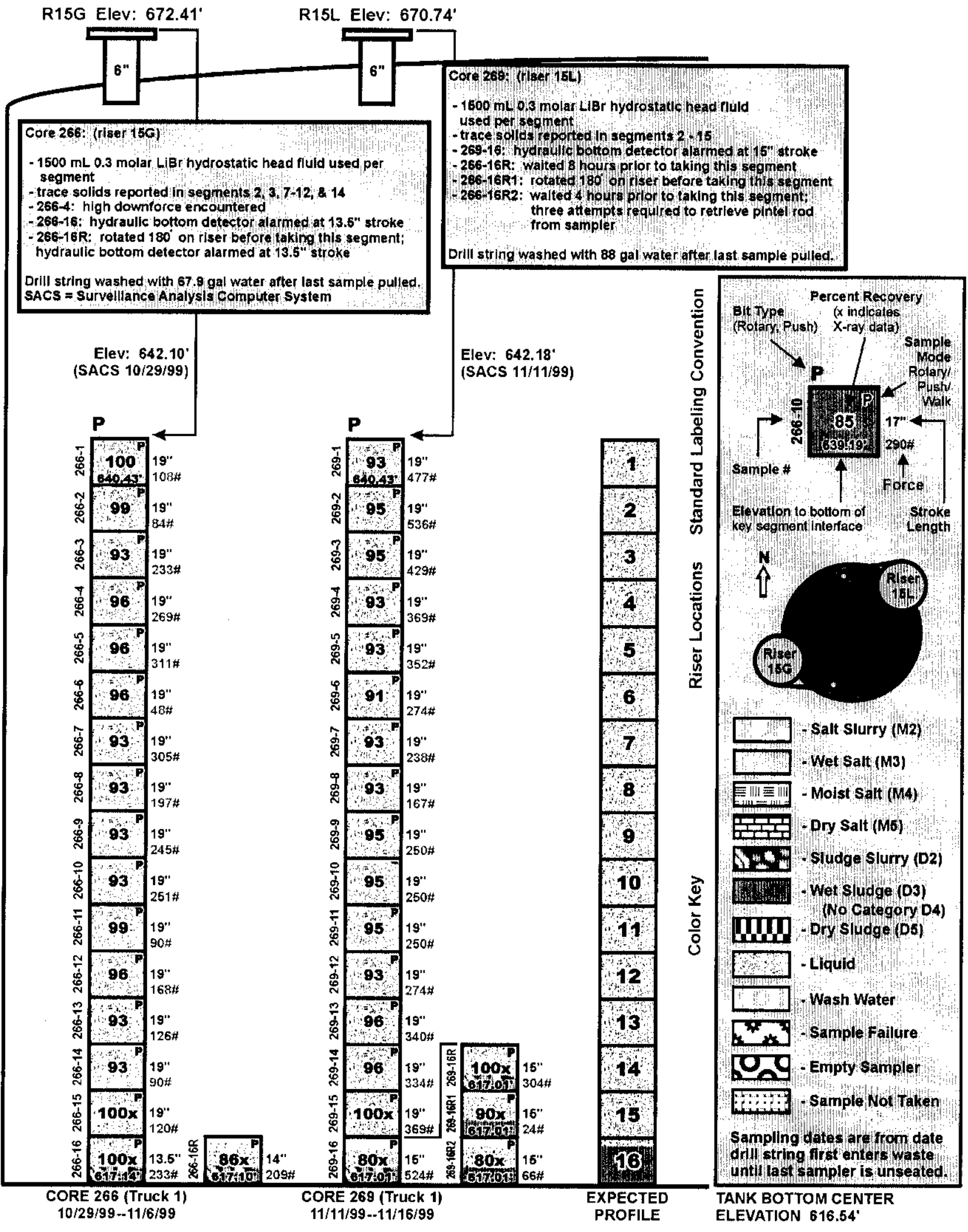




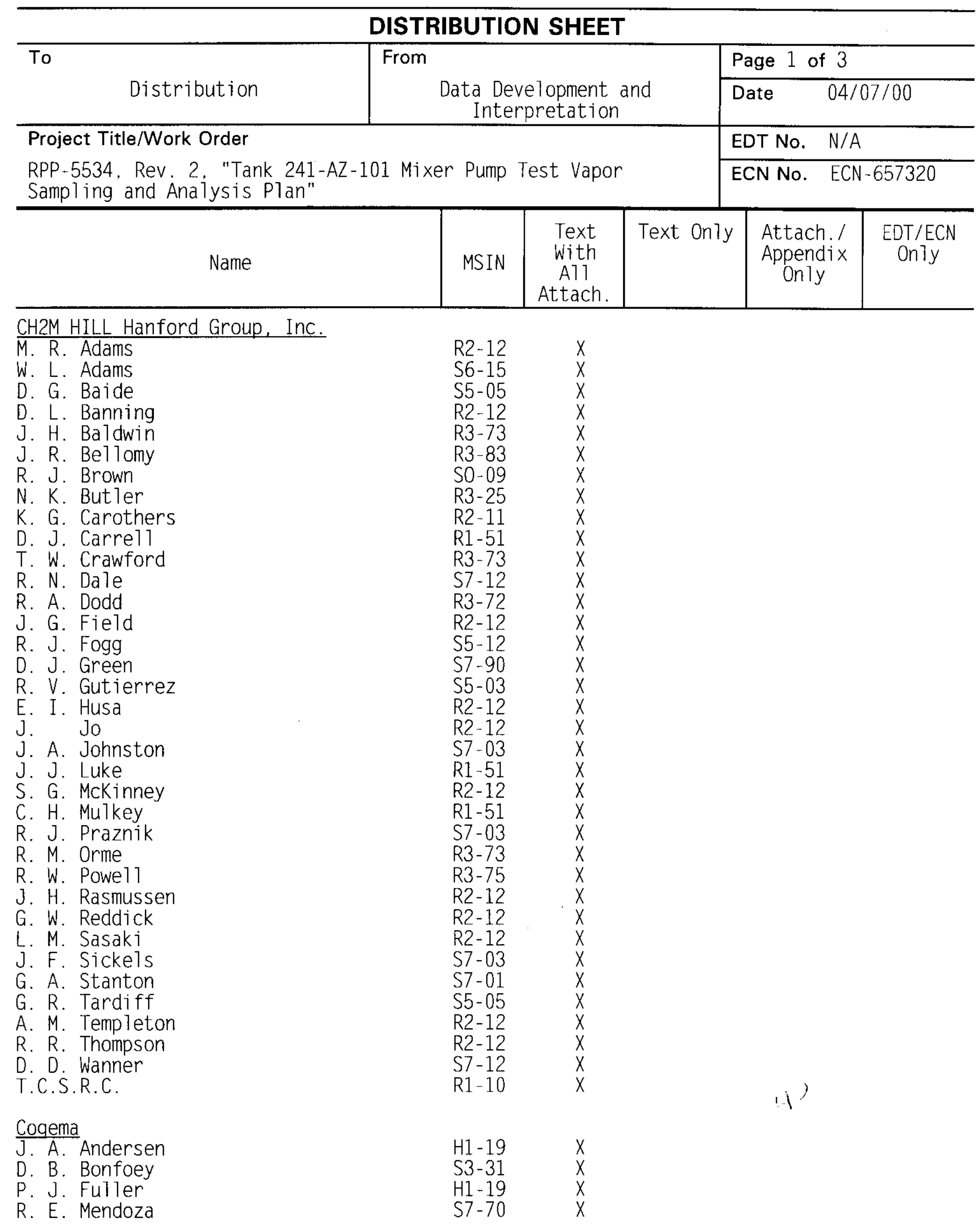


DISTRIBUTION SHEET

\begin{tabular}{|c|c|c|c|c|c|}
\hline \multirow[t]{2}{*}{ To } & \multirow{2}{*}{\multicolumn{3}{|c|}{ From }} & \multicolumn{2}{|c|}{ Page 2 of 3} \\
\hline & & & & Date & $04 / 07 / 00$ \\
\hline \multicolumn{4}{|l|}{ Project Title/Work Order } & \multicolumn{2}{|c|}{ EDT No. N/A } \\
\hline \multicolumn{4}{|c|}{$\begin{array}{l}\text { RPP-5534, Rev. 2, "Tank 241-AZ-101 Mixer Pump Test vapor } \\
\text { Sampling and Anaiysis Plan" }\end{array}$} & \multicolumn{2}{|c|}{ ECN No. $\quad$ ECN-657320 } \\
\hline Name & MSIN & $\begin{array}{c}\text { Text } \\
\text { with } \\
\text { All } \\
\text { Attach. }\end{array}$ & Text Only & $\begin{array}{l}\text { Attach. } \\
\text { Appendi } \\
\text { Only }\end{array}$ & $\begin{array}{l}\text { EDT/ECN } \\
\text { Only }\end{array}$ \\
\hline
\end{tabular}

Cogema - continued

L. A. Pingel

M. P. Rung

T. W. Staehr

M. Stauffer

Y. S. Tran

S3-31

S7 -70

R3-74

S3-31

H3-27

$x$

$x$

$x$

Fluor Hanford

R. Akita

K. E. Bell

L. A. Burchfield

S. L. Cobb

B. A. Crawford

R. A. Esch

R. K. Fuller

K. J. Greenough

J. C. Person

K. L. Powel1

K. Sathyanarayana

F. H. Steen

R. S. Viswanath

T6-50

$\mathrm{T} 6-12$

T6-12

S3-28

T6-50

$\mathrm{T} 6-12$

T6- 12

S3-30

T6-07

T6-04

L6-35

T6-12

S3-30

$x$

Lockheed Martin Services, Inc.

Central Files

B1-07 $\quad X$

MACTEC

R. D. Potter

R3-73 $\quad X$

Numatec Hanford Corporation

A. B. Carlson

R3-73

R3-73

R3-73

A. F. Choho

T6-50

D. R. Hansen

D. L. Herting

R. A. Kirkbride

W. I. Winters

T6-07

R3-73

T6-07

Office of River Protection

W. Abduhl

H6-60

H6-60

H6-60

W. Liou

J. A. Poppiti

DOE Reading Room

H6-60

H2-53

$x$
$x$
$x$
$x$
$x$
$x$
$x$
$x$
$x$
$x$
$x$
$x$
$x$ 


\section{DISTRIBUTION SHEET}

\begin{tabular}{|c|c|c|c|c|c|}
\hline \multirow{2}{*}{ To $\quad$ Distribution } & \multirow{2}{*}{\multicolumn{3}{|c|}{$\begin{array}{l}\text { From } \\
\text { Data Development and } \\
\text { Interpretation }\end{array}$}} & \multicolumn{2}{|l|}{ Page 3 of 3} \\
\hline & & & & \multicolumn{2}{|l|}{ Date } \\
\hline \multicolumn{4}{|l|}{ Project Title/Work Order } & \multicolumn{2}{|c|}{ EDT No. $N / A$} \\
\hline \multicolumn{4}{|c|}{$\begin{array}{l}\text { RPP-5534, Rev. 2, "Tank 241-AZ-101 Mixer Pump Test Vapor } \\
\text { Sampling and Analysis Plan" }\end{array}$} & \multicolumn{2}{|c|}{ ECN No. $\quad$ ECN-657320 } \\
\hline Name & MSIN & $\begin{array}{c}\text { Text } \\
\text { With } \\
\text { All } \\
\text { Attach. }\end{array}$ & Text Only & $\begin{array}{l}\text { Attach. / } \\
\text { Appendix } \\
\text { Only }\end{array}$ & $\begin{array}{l}\text { EDT/ECN } \\
\text { Only }\end{array}$ \\
\hline
\end{tabular}

Pacific Northwest National Laboratory

I. E. Burgeson

J. L. Huckaby

M. J. Truex

K. D. Wiemers

P7 -25

K7 -15

K2-10

$\mathrm{H} 6-61$

Y. Onishi

K7 -15

$X$
$X$
$X$
$X$
$X$

Vista Research

D. G. Douglas

P. C. Oh.

H4-01

H4-01

$X$
$X$

Waste Management Federal Services of Hanford, Inc.

S. N. Bakhtiar

D. B. Hardy

T6-12

S3-30

C. M. Seidel

G1-32

$X$
$X$
$X$

